\title{
Stabilization in the braid groups II: Transversal simplicity of knots
}

\author{
JOAN S BIRMAN \\ William W MENASCO
}

\begin{abstract}
The main result of this paper is a negative answer to the question: are all transversal knot types transversally simple? An explicit infinite family of examples is given of closed 3-braids that define transversal knot types that are not transversally simple. The method of proof is topological and indirect.
\end{abstract}

57M25; 57M50, 53C15

\section{Introduction and description of results}

This paper is about knots which are transverse to the standard tight contact structure in $\mathbb{R}^{3}$, and about the ways in which topological information about braids can be used to learn new things about these 'transversal knots'. Our approach to contact topology had its origins in the foundational paper of D Bennequin [2]. The results in this paper are direct outgrowths of our work on the Markov Theorem without Stabilization (MTWS) [6].

A knot in oriented $\mathbb{R}^{3}$ is the image $X$ of an oriented circle $S^{1}$ under a smooth embedding $e: S^{1} \rightarrow \mathbb{R}^{3}$. Viewing $S^{3}$ as $\mathbb{R}^{3} \cup \infty$, we also can think of $X$ as being a knot in $S^{3}$. The topological knot type $\mathcal{X}$ of $X$ is its equivalence class under smooth isotopy of the pair $\left(X, S^{3}\right)$. A representative $X \in \mathcal{X}$ is said to be a closed braid if there is an unknotted curve $\mathbf{A} \subset\left(S^{3} \backslash X\right)$, the axis, and a choice of fibration $\mathbf{H}$ of the open solid torus $S^{3} \backslash \mathbf{A}$ by meridian discs $\left\{H_{\theta} ; \theta \in[0,2 \pi)\right\}$ which we call fibers, such that $X$ intersects every disc fiber $H_{\theta}$ transversally. We call the pair $(\mathbf{H}, \mathbf{A})$ a braid structure on $\mathbb{R}^{3}$. If we regard $\mathbf{A}$ as the $z$-axis in $\mathbb{R}^{3}$ and the fibers of $\mathbf{H}$ as half-planes through $\mathbf{A}$, there are natural cylindrical coordinates $(\rho, \theta, z), \theta \in[0,2 \pi)$ in $\mathbb{R}^{3}$. Parameterizing $X$ by $(\rho(t), \theta(t), z(t)), t \in[0,2 \pi), X$ will be a closed braid if $\rho(t)>0$ and $d \theta / d t>0$ at every point $(\rho(t), \theta(t), z(t)) \in X$. See Figure 1(a), where two half-planes and two fragments of a closed braid $X$ are depicted. A braid isotopy of a closed braid is an isotopy that is transverse to the braid fibration and in the complement of the axis. The braid isotopy type of the closed braid $X$ is its equivalence class under braid isotopy. 


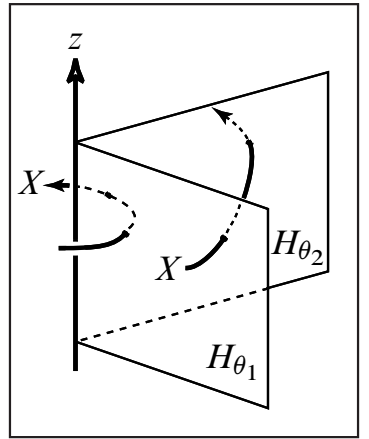

(a)

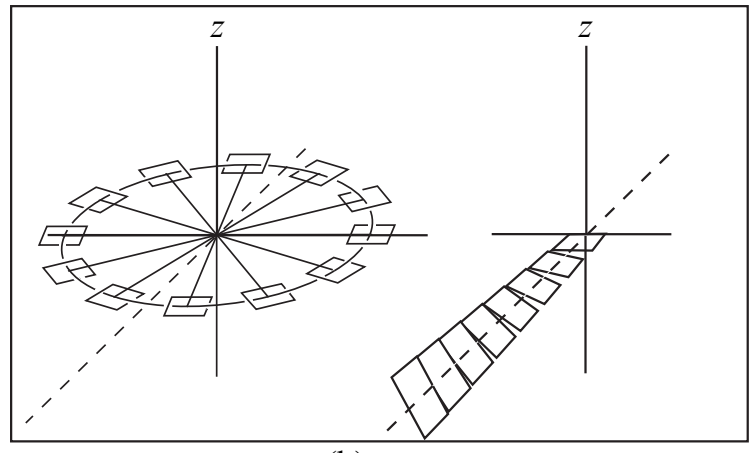

(b)

Figure 1: (a) Braid structure on $\mathbb{R}^{3}$; (b) 2-planes in the standard contact structure on $\mathbb{R}^{3}$. Note that these 2-planes are invariant under rotation about $z$-axis and translation along the $z$-axis (which we use as the braid axis $\mathbf{A}$ ).

Using the same cylindrical coordinates, the standard tight contact structure $\xi$ in oriented $\mathbb{R}^{3}$ is the kernel of the 1 -form $\alpha=\rho^{2} d \theta+d z$. Thinking of $\xi$ as attaching a 2-plane to each point in $\mathbb{R}^{3}$, Figure $1(\mathrm{~b})$ shows some of the 2 -planes associated to $\alpha$, for comparison of the braid structure and the contact structure. The field of 2-planes so-obtained is nowhere integrable.

An oriented knot $X$ in contact $\mathbb{R}^{3}$ is said to be transverse if it is everywhere transverse to the plane field of the standard contact structure. Parameterizing $X$ as before, a closed braid $X$ is transverse if and only if $d \theta / d t>-z^{\prime}(t) /(\rho(t))^{2}$ for every $t \in[0,2 \pi]$. We use the symbol $T X$ to denote a transversal knot. (For most of our discussion $T X$ will be transversal because it will also be a braid with respect to the $z$-axis.) Define two transverse knots to be transversally isotopic if there is an isotopy $h: \mathbb{R}^{3} \times I \rightarrow \mathbb{R}^{3}$ such that $h_{s}(X)=h(X, s) \subset \mathbb{R}^{3}$ is transversal for every $s \in I$. The transversal knot type $\mathcal{T} \mathcal{X}$ of a transverse knot $T X$ is its equivalence class under transversal isotopy. An obvious invariant of a transverse knot type is its topological knot type $[\mathcal{T} \mathcal{X}]_{\text {top }}=\mathcal{X}$.

In his seminal paper [2], Bennequin showed that braids could be useful for the study of contact structures on $S^{3}$ or $\mathbb{R}^{3}$. Recently that idea was generalized by E Giroux [11], who showed that open book decompositions of arbitrary 3-manifolds $M^{3}$ might be equally important for the study of contact structures on other 3-manifolds. Since an open book structure on a 3-manifold generalizes our braid structures on $\mathbb{R}^{3}$, this suggests that our work in this paper could have applications to the study of tight contact structures on other 3-manifolds. Bennequin proved that every transversal knot is transversally isotopic to a closed braid, and he discovered a very important numerical invariant $\beta(\mathcal{T} \mathcal{X})$ (the Bennequin invariant) of transversal knot type which is natural in 
the braid setting. To compute it when $T X$ is also a closed braid having the $z$-axis as it axis $\mathbf{A}$, consider a regular projection of $T X$ onto the plane $z=0$. Let $e(T X)$ be the the algebraic crossing number of $T X$ at the pre-images of the double points in the projection. Let $b(T X)$ be the braid index of $T X$, ie, its geometric linking number with A. Then $\beta(\mathcal{T} \mathcal{X})=e(T X)-b(T X)$. Note that both $e(X)$ and $b(X)$ are invariants of braid isotopy, and therefore $\beta$ is well-defined on any closed braid, ie, the closed braid need not represent a transversal knot type, and invariant under braid isotopy. Using topological techniques Bennequin showed that for a fixed topological knot type $\mathcal{X}$, the integer $\beta$ has an upper but not a lower bound. While $\beta(X)$ is well-defined on closed braids, it is not an invariant of $\mathcal{X}$. Indeed, each topological knot type $\mathcal{X}$ includes infinitely many distinct transversal knot types $\mathcal{T} \mathcal{X}$.

Are there other invariants of $\mathcal{T} \mathcal{X}$ in addition to $[\mathcal{T} \mathcal{X}]_{\text {top }}$ and $\beta(\mathcal{T} \mathcal{X})$ ? A hint that the problem might turn out to be quite subtle was in the paper [10] by Fuchs and Tabachnikov, who proved that while ragbags filled with invariants of transversal knot types $\mathcal{T} \mathcal{X}$ exist, based upon the work of V.I. Arnold in [1], they are all determined by $[\mathcal{T} \mathcal{X}]_{\text {top }}$ and $\beta(\mathcal{T} \mathcal{X})$. Thus, the seemingly new invariants that many people had discovered by using Arnold's ideas were just a fancy way of encoding $[\mathcal{T} \mathcal{X}]_{\text {top }}$ and $\beta(\mathcal{T} \mathcal{X})$. This lead to a definition: a transversal knot type $\mathcal{T} \mathcal{X}$ is transversally simple if it is determined by $[\mathcal{T} \mathcal{X}]_{\text {top }}$ and $\beta(\mathcal{T} \mathcal{X})$.

In [7] purely topological techniques were used to give a purely topological restriction on $\mathcal{X}$ which would insure that any associated $\mathcal{T} \mathcal{X}$ is transversally simple. This paper begins where the work in [7] ended. Our main result is to answer the question of whether all transversal knot types are transversally simple in the negative, by giving an explicit infinite family of examples of closed 3-braids that define transversal knot types that are not transversally simple. Our methods are topological and indirect and utilize the main result in [6]. After a draft of this paper was posted and submitted for publication, Etnyre and Honda proved the existence of examples, although they could not make them explicit [9]. Their methods, very different from ours, are based upon techniques in contact topology.

Here is a guide to this paper. In Section 2.1 we discuss our terminology and state Theorem 1, a simplified version of the MTWS. The full statement, and the proof, are to be found in [6]. In Section 2 our work is directed toward the proof of Theorem 2, a version of Theorem 1 which holds in the special case of links that are 3-braids, in the transversal setting. In Theorem 3 of Section 3 we give our promised infinite sequence of pairs of closed 3-braids that define transversal knots types that are not transversally simple, and prove that they have the properties that we claim they have. 
Acknowledgments We thank Keiko Kawamuro for her careful reading of both [6] and this paper. We also thank Hiroshi Matsuda for his very careful reading of an earlier version of this paper, which turned up an error. We had claimed that a certain template had braid index 6, but he showed that it had a closed 5-braid representative. The example, and all results that depended upon it, have been removed.

The first author acknowledges partial support from the following sources: the US National Science Foundation, under Grants DMS-9402988, 9705019, 9973232 and 0405586. The second author acknowledges partial support from the following sources: the U.S. National Science Foundations, under grants DMS-9200881, DMS-9626884 and DMS-0306062 ; and the Mathematical Sciences Research Institute, where he was a Visiting Member during winter/spring of 1997.

\section{The MTWS and transversal isotopies}

The goal of this section is to prove Theorem 2, a special and limited version of Theorem 1 , the main result in [6]. Theorem 1 is valid in the topological setting, whereas Theorem 2 is valid in the transversal setting.

In Section 2.1 our goal is to review enough of the background so that we can state Theorem 1. Its proof is in [6]. After that, we will redo some parts of the proof which were given in [6] in a transversal setting, with the goal of proving a version of the same theorem which applies to transversal knots. That work begins in Section 2.2, by redoing the 'basic construction' of Section 2.1 of [6] in the transversal setting. This construction gives us an immersed annulus which we call a 'transversal clasp annulus'. In Section 2.3 we review the basic ideas about the braid foliations which were the principle tool in [6], and use them to superimpose a braid foliation on the characteristic foliation which we obtained in Section 2.2 for the transversal clasp annulus. In Section 2.4 we use the work in Section 2.2 and 2.3 to state and prove Theorem 2.

Our goal in writing up this part of the paper has been to make it possible for readers who are interested mainly in the applications to be able to understand everything except parts of the proof of Theorem 2 .

\subsection{Block-strand diagrams, templates and the MTWS}

Let $\mathcal{B}$ be the collection of all braid isotopy classes of closed braid representatives of oriented knot types in oriented $\mathbb{R}^{3}$. Among these, consider the sub-collection $\mathcal{B}(\mathcal{X})$ of representatives of a fixed knot type $\mathcal{X}$. Among these, let $\mathcal{B}_{\text {min }}(\mathcal{X})$ be the sub-collection of representatives whose braid index is equal to the braid index of $\mathcal{X}$. Choose any 
$X_{+} \in \mathcal{B}(\mathcal{X})$ and any $X_{-} \in \mathcal{B}_{\min }(\mathcal{X})$. The classical Markov Theorem (see [5] for a proof which is in the spirit of the work in this paper) asserts that there exists a sequence of closed braids in $\mathcal{B}(\mathcal{X})$ :

$$
X_{+}=X_{1} \rightarrow X_{2} \rightarrow \cdots \rightarrow X_{r}=X_{-}
$$

such that, up to braid isotopy, each $X_{i+1}$ is obtained from $X_{i}$ by a single stabilization or destabilization. We call such a sequence a Markov tower. The existence of Markov towers in the transversal setting was proved by Orevkov and Shevchisin in [15]. In the transversal setting the stabilizations and destabilizations are all required to be positive. That is, each $X_{i+1}$ is obtained from $X_{i}$ by braid isotopy and a single positive destabilization (see Figure 2) or stabilization, where stabilization and destabilization are mutually inverse moves. The main result in [6] is to find such a sequence of closed braids, like the one in (1) relating $X_{+}$to $X_{-}$, but not using stabilizations, so that the braid index is non-increasing. This is done at the expense of introducing additional moves which are non-increasing on braid index.

We are interested in pairs of 'block-strand diagrams' which we call 'templates' (see Section 5.5, [6]). The concepts are intuitive and familiar, and the reader is referred to Figures 2, 3 and 4 for examples.

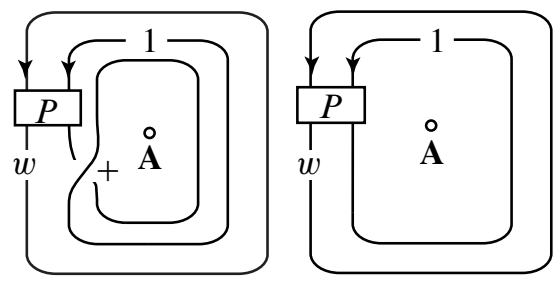

positive destabilization template

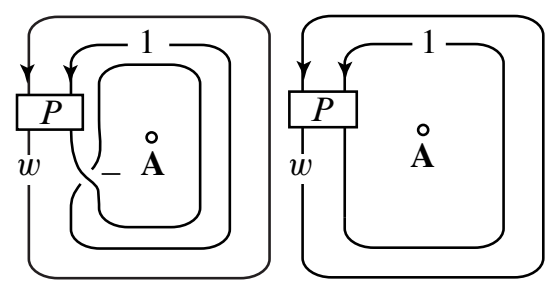

negative destabilization template

Figure 2: The two destabilization templates. Each template is a pair of block-strand diagrams.

A block $B$ in $\mathbb{R}^{3}-\mathbf{A}$ is a 3 -ball having the structure of a 2 -disk $\Delta$ crossed with an interval $[0,1]$ such that: (i) for any fiber $H_{\theta} \in \mathbf{H}$ the intersection $H_{\theta} \cap B$ is either $\varnothing$ or $\Delta \times\{\theta\}$ for some $\theta \in\left[\theta_{1}, \theta_{2}\right]$ and, (ii) there exists some $\theta \in[0,2 \pi)$ such that $H_{\theta} \cap B=\varnothing$. The disc $\mathbf{t}=B \cap H_{\theta_{1}}$ is the top of $B$ and the disc $\mathbf{B}=B \cap H_{\theta_{2}}$ is the bottom of $B$. A strand $l$ is homeomorphic to an interval $[0,1]$ or a circle $S^{1}$. It is oriented and transverse to each fiber of $\mathbf{H}$ such that its orientation agrees with the forward direction of $\mathbf{H}$. When $l$ is homeomorphic to an interval, $\partial l=l_{0} \cup l_{1}$, where $l_{0}$ is the beginning endpoint of $l$ and $l_{1}$ is the ending endpoint of $l$. 


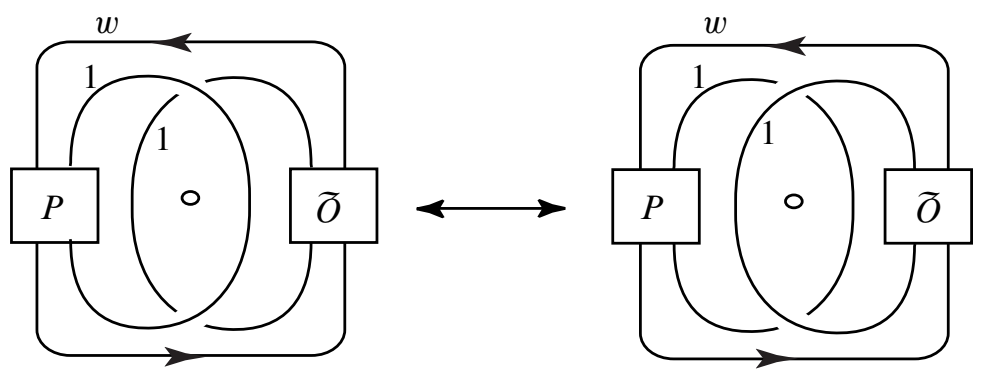

Figure 3: The exchange move template, $w \geq 2$, braid index $m \geq 4$

A block-strand diagram $\mathcal{D}$ is a collection of pairwise disjoint blocks $\left\{B^{1}, \cdots, B^{\mathrm{k}}\right\}$ and pairwise disjoint strands $\left\{l^{1}, \cdots, l^{1}\right\}$ which together have the following structure:

(i) If $l^{i} \cap B^{j} \neq \varnothing$ then $l^{i} \cap B^{j}=\left(l_{1}^{i} \cap \mathbf{t}^{j}\right) \cup\left(l_{0}^{i} \cap \mathbf{B}^{j}\right)$ where $\mathbf{t}^{j}$ is the top of $B^{j}$ and $\mathbf{B}^{j}$ is the bottom of $B^{j}$. (We allow for the possibility that either $\left(l_{1}^{i} \cap \mathbf{t}^{j}\right.$ ) or $\left(l_{0}^{i} \cap \mathbf{B}^{j}\right)$ is empty.)

(ii) For each $l_{0}^{i}$ (resp. $l_{1}^{i}$ ) there is some $\mathbf{B}^{j} \subset B^{j}$ (resp. $\mathbf{t}^{j} \subset B^{j}$ ) such that $l_{0}^{i} \subset \mathbf{B}^{j}$ (resp. $l_{1}^{i} \subset \mathbf{t}^{j}$ ).

(iii) For each block $B^{j}$ we have $\left|\mathbf{t}^{j} \cap\left(\cup_{1 \leq i \leq 1} l_{1}^{i}\right)\right|=\left|\mathbf{B}^{j} \cap\left(\cup_{1 \leq i \leq 1} l_{0}^{i}\right)\right| \geq 2$.

The fact that for each $j=1, \ldots, k$ there is a fiber which misses $B^{j}$ shows that, by rescaling, we may find a distinguished fiber $H_{\theta_{0}}$ which does not intersect any block. We define the braid index $b(\mathcal{D})$ of the block-strand diagram $\mathcal{D}$ to be the number of times the strands of $\mathcal{D}$ intersect the distinguished fiber $H_{\theta_{0}}$. Condition (iii) above makes $b(\mathcal{D})$ well defined.

A braiding assignment to a block-strand diagram $\mathcal{D}$ is a choice of a braid on $m_{j}$ strands for each $B^{j} \in \mathcal{D}$. That is, we replace $B^{j}$ with the chosen braid, so that $B^{j}$ with this braiding assignment becomes a braided tangle with $m_{j}$ in-strands and $m_{j}$ out-strands. In this way a block strand diagram with braiding assignments gives us a closed braid representative $X \subset \mathcal{D}$ of a link type $\mathcal{X}$. We say that $X$ is carried by $\mathcal{D}$.

A template $\mathcal{T}$ is a pair of block-strand diagrams $\left(\mathcal{D}_{+}, \mathcal{D}_{-}\right)$, both with blocks $B^{1}, \ldots, B^{\mathrm{k}}$ and an isotopy which takes $\mathcal{D}_{+}$to $\mathcal{D}_{-}$. That is, the blocks of $\mathcal{D}_{+}$are taken onto the blocks of $\mathcal{D}_{-}$and the strands of $\mathcal{D}_{+}$are taken onto the strands of $\mathcal{D}_{-}$. The diagrams $\mathcal{D}_{+}$and $\mathcal{D}_{-}$are the initial and final block-strand diagrams in the pair. Thus, for every fixed choice of braiding assignments to the blocks $B^{1}, \ldots, B^{\mathrm{k}}$ the resulting closed braids $X_{+} \subset \mathcal{D}_{+}$and $X_{-} \subset \mathcal{D}_{-}$represent the same oriented link type $\mathcal{X}$; and the isotopy from $\mathcal{D}_{+}$to $\mathcal{D}_{-}$induces an isotopy from $X_{+}$to $X_{-}$. 
We use the notation $\mathcal{T}(m, n)$ for the collection of all topological templates whose $\mathcal{D}_{+}$ (resp. $\mathcal{D}_{-}$) has braid index $m$ (resp. $n$ ), where $m \geq n$. Readers who are familiar with [6] will note that we have changed the definition of $\mathcal{T}(m, n)$ slightly from the definition given in Theorem 2 of [6], by including destabilizations, exchange moves and flypes in $T(m, n)$. We did it because it makes the statement more concise; also we will not be concerned here, as we were in [6], with the actual construction of the templates, so there is no reason to single out some templates and treat them separately from others.

Examples of templates are the block-strand diagram pairs which make up the templates in Figure 2, 3 and 4. The strands may carry positive integer weights, where weight $w$ means replace the strand by $w$ parallel strands in the given projection.

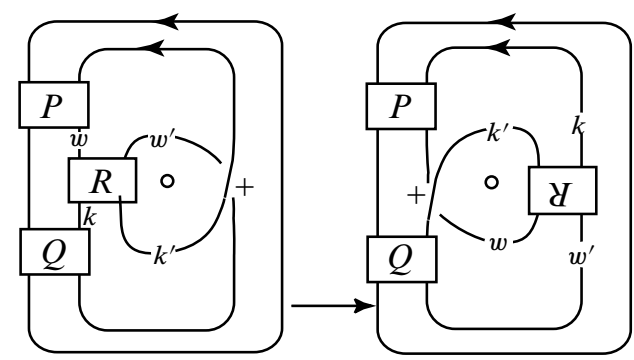

positive destabilization template

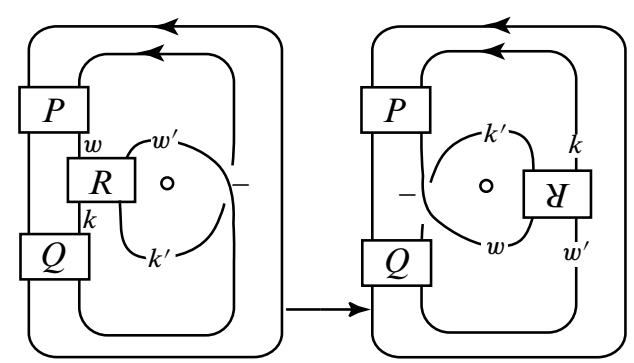

negative destabilization template

Figure 4: The two flype templates. The weights must satisfy $k^{\prime}-w \geq 0$. Note that the device of weighted strands may conceal half-twists introduced during the flype. See Figure 4 of [6].

There are restrictions on the weights. The exchange move template of Figure 3 has braid index $m=w+2$, so that it appears in every $\mathcal{T}(m, n)$ with $m \geq 3$. However, it can be replaced by braid isotopy if $m=n=3$ (except in the special case of composite knots of braid index 3 ), so we assume that $w \geq 2$. As for the flype template of Figure 4 the restriction on the weights is needed to insure that the flype is non-increasing on braid index.

A braid $X$ is said to be exchange equivalent to $X^{\prime}$ if there exists an isotopy taking $X$ to $X^{\prime}$ that is a sequence of braid isotopies and exchange moves. We say $X$ is exchange reducible to $X^{\prime}$ if there exists an isotopy taking $X$ to $X^{\prime}$ that is a sequence of braid isotopies, exchange moves and destabilizations.

We are now ready to state the main result in [6], the MTWS. We have simplified the statement, because we do not need the full strength of the theorem proved in [6] for present purposes. 
Theorem 1 (Markov's Theorem Without Stabilization [6]) Let $\mathcal{X}$ be an oriented knot type and let $\left(X_{+}, X_{-}\right)$be a pair of closed braids such that $X_{-} \in \mathcal{B}_{\min }(\mathcal{X})$ and $X_{+} \in \mathcal{B}(\mathcal{X})$ with $b\left(X_{+}\right)=m$ and $b\left(X_{-}\right)=n$. Then there exist $X_{+}^{\prime} \in \mathcal{B}(\mathcal{X}), X_{-}^{\prime} \in$ $\mathcal{B}_{\min }(\mathcal{X})$, and a finite set of templates $\mathcal{T}(m, n)$ (whose membership is only dependent on $(m, n))$ such that:

(1) $X_{+}$is exchange reducible to $X_{+}^{\prime}$ and $X_{-}$is exchange equivalent to $X_{-}^{\prime}$, and

(2) There is a template $\left(\mathcal{D}_{+}, \mathcal{D}_{-}\right)$in the finite set $\mathcal{T}(m, n)$ where, using the same braiding assignment, on $\mathcal{D}_{+}$and $\mathcal{D}_{-}$, the diagram $\mathcal{D}_{+}$carries $X_{+}^{\prime}$ and the diagram $\mathcal{D}_{-}$carries $X_{-}^{\prime}$.

\subsection{The construction of transversal clasp annuli}

The goal in this subsection and the two that follow it is to state and prove a modified version of Theorem 1 which holds in the transversal setting. The proof of the MTWS in [6] began with the construction of a 'clasp annulus'. We were given closed braids $X_{+}$and $X_{-}$which represent the same topological knot type $\mathcal{X}$. The goal was to find an isotopy from $X_{+} \rightarrow X_{-}$through closed braids, which would give us moves that change the closed braid, but were non-increasing on braid index. It was proved in Section 2.1 of [6], that the trace of the isotopy from $X_{+}$to $X_{-}$could be assumed to be a clasp annulus, that is a topological annulus $\mathcal{P A} \cong S^{1} \times[0,1]$ with boundary and an immersion $e: \mathcal{P A} \longrightarrow \mathcal{C A} \subset \mathbb{R}^{3}$ such that:

- The boundary of $C A$ is $X_{+}-X_{-}$. The self-intersection set of $\mathcal{C A}$ is the union of clasp $\operatorname{arcs}\left\{\gamma^{1}, \cdots, \gamma^{\mathrm{k}}\right\} \subset \mathcal{C A}$ such that, for each $\gamma^{j}$, we have $e^{-1}\left(\gamma^{j}\right)=$ $\gamma_{+}^{j} \cup \gamma_{-}^{j} \subset \mathcal{P A}$. Also, $\gamma_{\epsilon}^{j}$ has an endpoint on $e^{-1}\left(X_{\epsilon}\right), \epsilon= \pm$. In particular, there are no ribbon arcs or other more complicated self-intersections.

We refer to $\mathcal{P A}$ as the pre-image annulus (cf Section 2.1, [6]). The motion of pushing $X_{+}$across $\mathcal{C A}$ to $X_{-}$corresponds to an isotopy in $\mathbb{R}^{3}$. The clasp annulus $\mathcal{C A}$ can be positioned with respect to the braid structure $(\mathbf{H}, \mathbf{A})$ so as to have a 'braid foliation' (see Section 2.3 below), that encodes a Markov tower for the pair $\left(X_{+}, X_{-}\right)$. We called this our basic construction.

In this subsection we adapt the same ideas, but now in the setting of the standard contact structure and transversal isotopies in $\mathbb{R}^{3}$, and construct our transversal clasp annulus. The construction here was motivated by, but does not depend on, that in [6].

We will need to make repeated use of two results which are well-known to workers in low dimensional contact topology. In [2], Bennequin proved that a transversal link in the standard contact structure of $\mathbb{R}^{3}$ could be modified by transversal isotopy to a transversal link which is in braid position with respect to the $z$-axis. In fact, it is easy to see that Bennequin's original argument generalizes to the following statement. 
Lemma 1 (The Bennequin Trick) Let $\Gamma \subset \mathbb{R}^{3}$ be an oriented differentiable graph that is transversal to the standard contact structure $\xi$ of $\mathbb{R}^{3}$. Let $e \subset \Gamma$ be an edge having a local parameterization function $f(t):[0,1] \rightarrow \mathbb{R}^{3}$ where $f(t)=(\rho(t), \theta(t), z(t))$. If $\frac{d \theta}{d t}$ is not positive for all $t$ then there exists a transversal isotopy of $\Gamma$ which is the identity everywhere except on a small neighborhood of $e$ such that for a resulting parameterization $\widehat{f}(t)=(\hat{\rho}(t), \hat{\theta}(t), \widehat{z}(t))$ we have $\frac{d \hat{\theta}}{d t}$ being everywhere positive.

The Bennequin trick is analogous to the Alexander trick, which was used in Section 2.1 of [6]. It essentially allows us to put a transversal graph into braid position, ie, $\Gamma \cap \mathbf{A}=\varnothing$ and $\frac{d \theta}{d t}>0$ for every edge in $\Gamma$. The Bennequin trick will be used in conjunction with a result of Eliashberg, which allows us to extend a transversal isotopy of links to an isotopy of the ambient space $\mathbb{R}^{3}$, with its standard tight contact structure.

Lemma 2 (Eliashberg's isotopy extension lemma [8]) Let $\varphi_{t}: X \rightarrow \mathbb{R}^{3}, t \in[0,1]$ be an transversal isotopy of a transversal link in the standard contact structure $\xi$. Then there exists a contact isotopy $\alpha_{t}:\left(\mathbb{R}^{3}, \xi\right) \rightarrow\left(\mathbb{R}^{3}, \xi\right), t \in[0,1]$, with $\alpha_{0}=i d$ such that $\alpha_{t} \circ \varphi_{0}=\varphi_{t}$ for $t \in[0,1]$.

With the help of these two lemmas we will now develop the construction of Section 2.1 of [6] in the contact structure setting. Figure 5 may be helpful.

Given a pair $\left(T X_{+}, T X_{-}\right)$with $T X_{+}, T X_{-} \in \mathcal{T} \mathcal{X}$ and $\left[T X_{+}\right]_{\text {top }},\left[T X_{-}\right]_{\text {top }} \in \mathcal{B}(\mathcal{X})$, $\mathcal{T A}$ is an associated transversal clasp annulus if for $\mathcal{P A} \cong S^{1} \times[0,1]$, there exists an immersion $e: \mathcal{P A} \longrightarrow \mathcal{T A} \subset \mathbb{R}^{3}$, with the oriented boundary of $\mathcal{T A}$ being $T X_{+}-T X_{-}$, such that:

(a) The self-intersection set of the immersed annulus $\mathcal{T A}$ is the union of clasp arcs $\left\{\gamma^{1}, \cdots, \gamma^{\mathrm{k}}\right\} \subset \mathcal{T A}$ such that, for each $\gamma^{j}$, we have $e^{-1}\left(\gamma^{j}\right)=\gamma_{+}^{j} \cup \gamma_{-}^{j}$. Also, $\gamma_{\epsilon}^{j}$ has an endpoint on $e^{-1}\left(T X_{\epsilon}\right), \epsilon= \pm$.

(b) The characteristic foliation of $\mathcal{T A}$ (which comes from integrating $\xi$ in $\mathcal{T A}$ ) is trivial. That is, when viewing the pre-image of a leaf of the characteristic foliation in $\mathcal{P A}$, it is a single arc having one endpoint on $e^{-1}\left(T X_{+}\right)$and one endpoint on $e^{-1}\left(T X_{-}\right)$.

(c) The clasp arcs $\left\{\gamma^{1}, \cdots, \gamma^{\mathrm{k}}\right\}$ are transversal arcs in the contact structure. In particular, the arcs $\gamma_{+}^{i}, \gamma_{-}^{i} \subset \mathcal{P A}$ are transverse to the pre-image of the characteristic foliation of $\mathcal{T} \mathcal{A}$. See Figure 5(b) for an example. Each clasp arc is also in braid position, ie, transverse to each fiber $H_{\theta}$ of the braid structure $\mathbf{H}$ on $\mathbb{R}^{3}$. Here we assume that the braid axis is the $z$ axis. 
(d) There exists a disjoint set of extension arcs of the clasp arcs (Figure 5(c)), $\left\{g_{\epsilon}^{1}, \cdots, g_{\epsilon}^{\mathrm{k}}, \epsilon= \pm\right\} \subset \mathcal{P} \mathcal{A}$, such that for each $i=1, \ldots, k$ the union $g_{\epsilon}^{i} \cup \gamma_{\epsilon}$ is an continuous edgepath in $\mathcal{P A}$ that is transverse to the trivial foliation of $\mathcal{P A}$ and has its two endpoints on $e^{-1}\left(T X_{\epsilon}\right)$. Each extension arc is also in braid position.

(e) There exists a braid $T X_{0} \subset\left\{\mathcal{T A} \backslash\left(\cup_{1 \leq i \leq \mathrm{k}} \gamma^{i} \cup \cup_{1 \leq i \leq \mathrm{k}}\left(g_{+}^{i} \cup g_{-}^{i}\right)\right)\right\}$ such that $\left[T X_{0}\right]_{\text {top }} \in \mathcal{B}(\mathcal{X})$ and $\left[T X_{0}\right]_{\text {top }}$ is a preferred longitude of both $\left[T X_{+}\right]_{\text {top }}$ (in the absence of $\left.\left[T X_{-}\right]_{\text {top }}\right)$ and $\left[T X_{-}\right]_{\text {top }}$ (in the absence of $\left[T X_{+}\right]_{\text {top }}$ ).

(f) The graph

$$
T X_{+} \cup T X_{-} \cup T X_{0} \cup\left(\cup_{1 \leq i \leq \mathrm{k}} \gamma^{i}\right) \cup\left(\cup_{1 \leq i \leq \mathrm{k}} e\left(g_{+}^{i}\right)\right) \cup\left(\cup_{1 \leq i \leq \mathrm{k}} e\left(g_{-}^{i}\right)\right)
$$

is in braid position with respect to the $z$-axis.

(g) The collection of edgepaths $\left\{\left(g_{+}^{i} \cup \gamma_{+}^{i}\right),\left(g_{-}^{i} \cup \gamma_{-}^{i}\right), i=1, \ldots, k\right\} \subset \mathcal{P A}$ is staggered. That is, there exists a set of leaves $\left\{s^{1}, \cdots, s^{1}\right\} \subset \mathcal{P A}$ in the preimage of the characteristic foliation that are disjoint from the $g_{ \pm}^{i} \cup \gamma_{ \pm}^{i}$ edgepaths, $1 \leq i \leq \mathrm{k}$, such that each component of $\mathcal{P A} \backslash\left(\cup_{1 \leq j \leq 1} s^{j}\right)$ is a disc containing at most a single edgepath from the set $\left\{\left(g_{+}^{i} \cup \gamma_{+}^{i}\right),\left(g_{-}^{i} \cup \gamma_{-}^{i}\right), i=1, \ldots, k\right\}$.

We now use The Bennequin Trick (Lemma 1) and the Eliashberg Lemma (Lemma 2) to establish that for any such pair $\left(T X_{+}, T X_{-}\right)$there always exists a transversal clasp annulus.

Proposition 1 Given any pair of transversal closed braids $\left(T X_{+}, T X_{-}\right)$of the same transversal knot type, there exists an associated transversal clasp annulus $\mathcal{T A}$ with ว $\mathcal{T A}=T X_{+}-T X_{-}$.

Proof We start by partitioning $\mathbb{R}^{3}$ along the plane $z=0$, and denote the upper-half space by $\mathbb{R}_{+}^{3}$ and the lower-half space by $\mathbb{R}_{-}^{3}$. We position $T X_{-}$so that it is a transverse braid in $\mathbb{R}_{-}^{3}$. Let $T X^{\prime} \in \mathcal{T} \mathcal{X}$ be an oriented closed braid that is a preferred longitude of $T X_{-}$. We can visualize $T X^{\prime}$ being in $\mathbb{R}_{-}^{3}$, in braid position, and close to $T X_{-}$ with $T X^{\prime}-T X_{-}$the boundary of an embedded annulus $\mathcal{A}^{\prime}$ in the lower half-space $\mathbb{R}_{-}^{3}$ whose characteristic foliation is trivial. In Figure 5(a) we see two fragments of $\mathcal{A}^{\prime}$. Note that $\mathcal{A}^{\prime}$ is oriented by the orientation on its boundary curves, so that we are looking at its negative side. The knots $T X^{\prime}$ and $T X_{-}$will be geometrically linked, except in the special case when $\mathcal{T} \mathcal{X}$ is the unknot. Let $T X_{0} \subset \mathcal{A}^{\prime}$ be a core curve of the annulus. Clearly $T X_{0}$ is also a preferred longitude of $T X_{-}$and $T X^{\prime}$.

Now we think of a regular projection of the link $T X_{-} \sqcup T X^{\prime}$ onto the plane $z=0$. Viewing this projection from the $\mathbb{R}_{+}^{3}$ side, at any crossing of $T X_{-}$and $T X^{\prime}$, whenever 

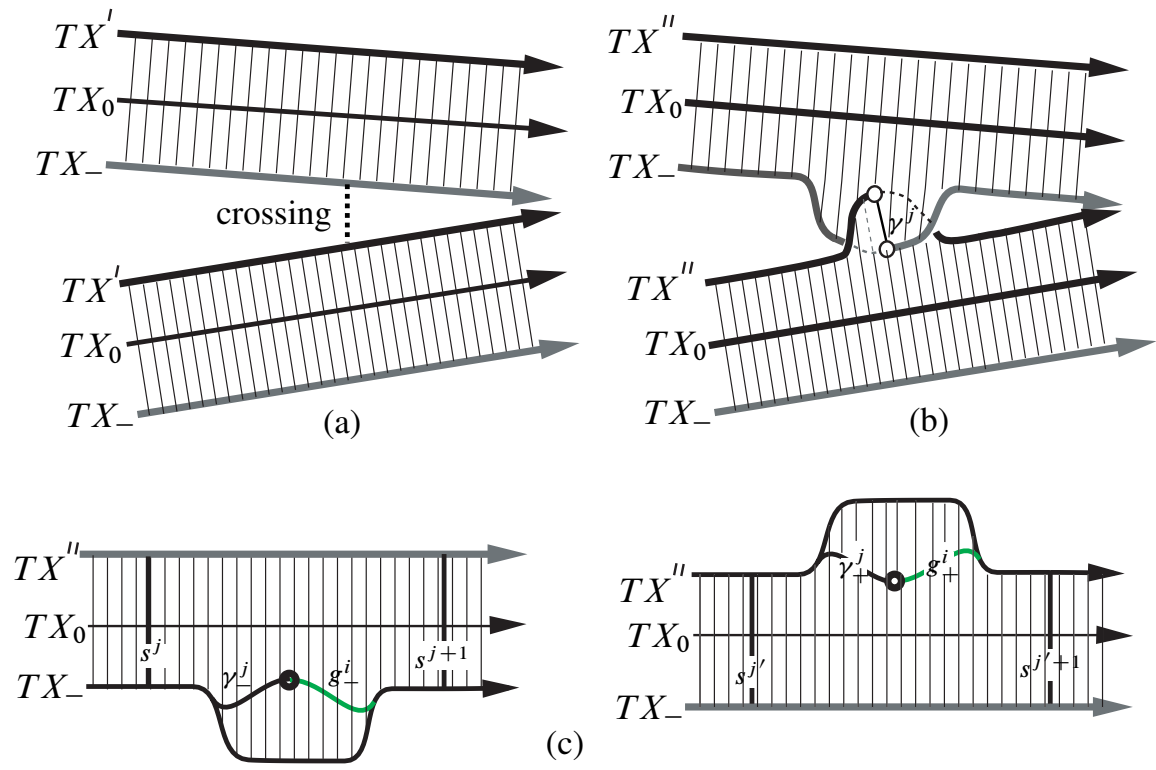

(c)

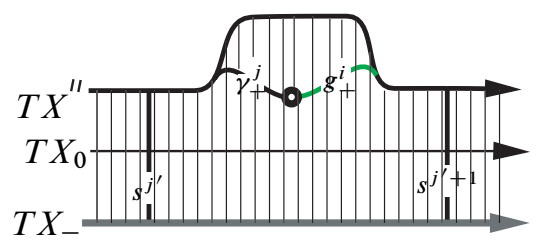

Figure 5: (a) Fragments of the embedded annulus $\mathcal{A}^{\prime}$, trivially foliated, with $\partial \mathcal{A}^{\prime}=\mathcal{T} \mathcal{X}^{\prime}-\mathcal{T} \mathcal{X}_{-}$. (b) The immersed annulus $\mathcal{A}^{\prime \prime}$, with $\partial \mathcal{A}^{\prime \prime}=$ $\mathcal{T} \mathcal{X}^{\prime \prime}-\mathcal{T} \mathcal{X}_{-}$. (c) The preimage of $\mathcal{A}^{\prime \prime}$. (Changing the label $T X^{\prime \prime}$ to $T X_{+}$, sketch (c) can also be interpreted as illustrating the preimage of $\mathcal{T A}$.)

$T X^{\prime}$ goes under $T X_{-}$we modify it by an isotopy of $T X^{\prime}$, changing the crossing. (We do not change any of the crossings between $T X^{\prime}$ and $T X_{0}$.) We would like to do this 'unlinking' transversally, and for that we use a method which was used by Nancy Wrinkle in her thesis [16]. She notes:

$\star \quad$ Let $C_{\rho}$ be a cylinder of radius $\rho$ in $\mathbb{R}^{3}$ whose axis is parallel to the $z$-axis and is foliated by parallel Legendrian arcs of slope $-\rho^{2}$. Then an isotopy of subarcs of a transverse braid which lies on the surface of $C_{\rho}$, and is slid vertically along the Legendrian leaves, is a transverse isotopy.

So, let $p=p_{i} \in \mathbb{R}^{2}$ be a double point in the projected image of $T X_{-} \cup T X^{\prime}$ onto $\mathbb{R}^{2}$, let $p_{i}^{\prime}$ be its preimage on $T X^{\prime}$ and let $\alpha_{i}$ be a neighborhood of $p_{i}^{\prime}$ on $T X^{\prime}$. For each $i$ the points $p_{i}$ and $p_{i}^{\prime}$ share the same $\rho$ coordinate $\rho_{i}$, so they lie on the cylinder $C_{\rho_{i}}$. Choose a leaf of the characteristic foliation of $C_{\rho_{i}}$ that passes through $p_{i}$ and shift the point $p_{i}^{\prime}$ a little bit until it also lies on the leaf. Then slide $\alpha_{i}$ (keeping its endpoints fixed) to an $\operatorname{arc} \beta_{i}$, thereby changing the crossing in $T X_{-} \cup T X^{\prime}$. Let $T X^{\prime \prime}$ be the transverse knot that results from repeating this procedure at each undercrossing of $T X^{\prime}$, replacing each $\alpha_{i}$ by $\beta_{i}$. 
Our unlinking isotopy will create clasp intersections in $\mathcal{A}^{\prime}$ as shown in Figure 5. We can assume that the clasp arcs are in braid position. We call the resulting annulus $\mathcal{A}^{\prime \prime}$. Its boundary will be $T X^{\prime \prime}-T X_{-}$. The characteristic foliation on $\mathcal{A}^{\prime \prime}$ is still trivial and the image of extension arcs can be easily chosen in $\mathcal{A}^{\prime \prime}$. Moreover, a set of leaves in the characteristic foliation can readily chosen to demonstrate that the edgepath of clasps and their extension are staggered. (See Figure 5.) Notice that $T X^{\prime \prime}$ and $T X_{-}$ are geometrically unlinked. So we can modify $T X^{\prime \prime}$ by transversal isotopy so that it is contained in $\mathbb{R}_{+}^{3}$. This transversal isotopy will also correspond to a braid isotopy in the complement of the $z$-axis, which is the braid axis. Finally, notice that for the pair $\left(X^{\prime \prime}, T X_{-}\right)$, the annulus $\mathcal{A}^{\prime \prime}$ is a transversal clasp annulus.

By Lemma 1 we know that closed oriented braids in $\mathbb{R}^{3}$ having the $z$-axis as their braid axis are transversally isotopic to transversal closed braids. We consider a pair $\left(T X_{+}, T X_{-}\right)$such that $\left[T X_{+}\right]_{\text {top }} \in \mathcal{B}(\mathcal{X}),\left[T X_{-}\right]_{\text {top }} \in \mathcal{B}_{\min }(\mathcal{X})$ and $T X_{+}, T X_{-} \in \mathcal{T} \mathcal{X}$. The main result in [15] tells us that there is a transversal Markov tower for the pair of transversal knots $\left(T X_{+}, T X_{-}\right)$. That is, the transversal isotopy may be assumed to be via a sequence of transverse closed braids, say:

$$
T X_{+}=T X_{1} \rightarrow T X_{2} \rightarrow \cdots \rightarrow T X_{r}=T X_{-},
$$

such that each $T X_{j+1}$ is obtained from $T X_{j}$ by braid isotopy and a positive stabilization or destabilization. Negative stabilizations and destabilizations do not occur.

Without loss of generality, we can assume that this isotopy is restricted to $\mathbb{R}_{+}^{3}$, ie, it is the identity on $\mathbb{R}_{-}^{3}$. By Lemma 2 our transversal isotopy via the Markov tower extends to a contact isotopy $\Psi_{0}:\left(\mathbb{R}^{3}, \xi\right) \rightarrow\left(\mathbb{R}^{3}, \xi\right)$, which fixes $\mathbb{R}_{-}^{3}$ and, thus $T X_{-}$. This is the starting point for our transversal clasp annulus. We initially let $\mathcal{T A}_{0}=\Psi_{0}\left(\mathcal{A}^{\prime \prime}\right)$. The boundary of $\mathcal{T} \mathcal{A}_{0}$ will then be $\Psi_{0}\left(T X^{\prime \prime}-T X_{-}\right)=T X_{+}-T X_{-}$. Note that since $\Psi_{0}$ is a contact isotopy the characteristic foliation of $\mathcal{T A}_{0}$ will be trivial, since the foliation on $\mathcal{A}^{\prime \prime}$ was trivial. Moreover, the fact that the clasp arc and their extensions are transverse and staggered in this trivial foliation is preserved by $\Psi_{0}$. But, we are not yet done. Although $T X_{+}$and $T X_{-}$are in braid position, we cannot be sure that $T X_{0}$ remains in braid position under $\Psi_{0}$, nor do we know the positioning of the clasp $\operatorname{arcs}\left\{\gamma^{1}, \cdots, \gamma^{\mathrm{k}}\right\}$, nor the image under the immersion of the extension arcs $\left\{e\left(g_{+}^{1}\right), e\left(g_{-}^{1}\right), \cdots, e\left(g_{+}^{\mathrm{k}}\right), e\left(g_{-}^{\mathrm{k}}\right)\right\}$. However, if they are not in braid position, we can apply the Bennequin trick (Lemma 1) to place them in braid position via a transversal isotopy of the graph

$$
T X_{+} \cup T X_{-} \cup T X_{0} \cup\left(\cup_{1 \leq i \leq \mathrm{k}} \gamma^{i}\right) \cup\left(\cup_{1 \leq i \leq \mathrm{k}} e\left(g_{+}^{i}\right)\right) \cup\left(\cup_{1 \leq i \leq \mathrm{k}} e\left(g_{-}^{i}\right)\right)
$$

which is the identity on $T X_{+}$and $T X_{-}$. Appealing again to Lemma 2, we extend this transversal isotopy to a contact isotopy $\Psi_{1}:\left(\mathbb{R}^{3}, \xi\right) \rightarrow\left(\mathbb{R}^{3}, \xi\right)$. Our needed transversal 
annulus associated with the pair $\left(T X_{+}, T X_{-}\right)$will be $\mathcal{T A}=\Psi_{1}\left(\mathcal{T A}_{0}\right)=\Psi_{1} \circ \Psi_{0}\left(\mathcal{A}^{\prime \prime}\right)$. Again, the triviality of the characteristic foliation of $\mathcal{A}^{\prime \prime}$ is preserved under contact isotopies. The fact that clasp arcs and their extensions are staggered remains true under $\Psi_{1}$. And, by construction $\mathcal{T A}$ satisfies the other conditions of a transversal clasp annulus.

\subsection{Braid foliations on transversal clasp annuli}

The principle tool in the proof of the MTWS is the study of the braid foliation of $\mathcal{C A}$, the singular foliation which is determined by the intersections of $\mathcal{C A}$ with the disc fibers $H_{\theta}$ of $\mathbf{H}$. Braid foliations have been reviewed in several places. We refer readers who are unfamiliar with them to the review in Sections 3 and 4 of [6], and if necessary thence to [3] for additional details. However, there are aspects of these foliations which will be needed here and may not be so familiar, so we recall them next. After that, we will prove Proposition 2, which describes the braid foliation on the transversal clasp annulus of the preceding section.
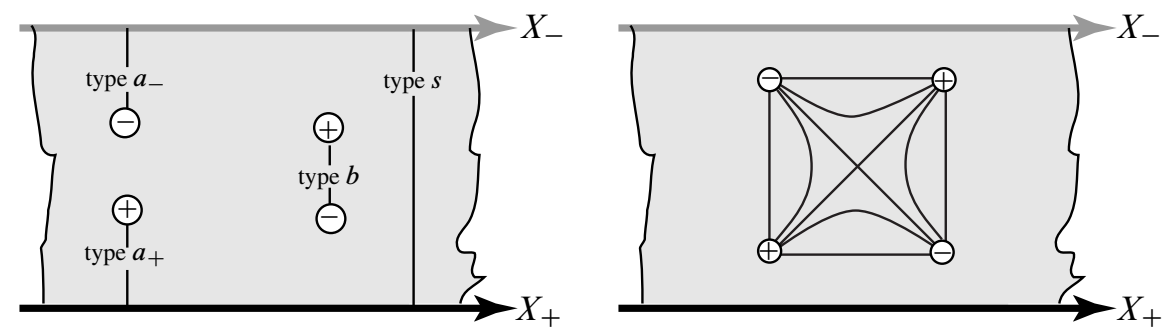

Figure 6: The left sketch illustrates the types of non-singular leaves in the foliation of $\mathcal{C A}$. The right sketch illustrates a foliated neighborhood of a type $b b-$ singularity.

As in [6] and [3]: Given a braid structure $(\mathbf{H}, \mathbf{A})$ and a clasp annulus $\mathcal{C A}$, we arrange that $\mathbf{A}$ transversally intersects $\mathcal{C A}$ in finite many points. The points of $\mathcal{C A} \cap \mathbf{A}$ are the vertices of the braid foliation. Since $\mathcal{C A}$ has an orientation that is consistent with the orientation of $X_{+}$, and since $\mathbf{A}$ also has an orientation, each vertex of the braid foliation will have an associated parity. We can also assume that all but finitely many disc fibers of $H_{\theta} \in \mathbf{H}$ will intersect $\mathcal{C A}$ transversally. Of the finitely many disc fibers that do not intersect $\mathcal{C A}$ transversally, each contains a single point of non-transversality that corresponds to a saddle point. Since $\mathcal{C A}$ is oriented, and since the orientations on $\mathbf{A}$ and the fibers of $\mathbf{H}$ are consistent, there is also a natural parity assignment to each singularity in the braid foliation of $\mathcal{C A}$. (See the left sketch in Figure 6, and see [6] and in particular [3] for all details). 
After an isotopy of $\mathcal{C A}$ with respect to the braid structure $(\mathbf{H}, \mathbf{A})$ we may assume that the non-singular leaves of the braid foliation on $\mathcal{C A}$ are either $s-\operatorname{arcs}, a-\operatorname{arcs}$ or $b$-arcs. The $a$-arcs subdivide into two groups: $a_{+}-\operatorname{arcs}$ which have one endpoint on a positive vertex and one endpoint on $X_{+}$; and $a_{-}$-arcs which have one endpoint on a negative vertex and one endpoint on $X_{-}$. In the foliation the set of possible types of singularities can be listed in correspondence to the types of leaves that are in a regular neighborhood of the singularities. Such a regular neighborhood is called a tile. For example, the right sketch in Figure 6 illustrates a foliated $b b$-tile.

In Figure 7 we show the listing of possible singularity and tile types.
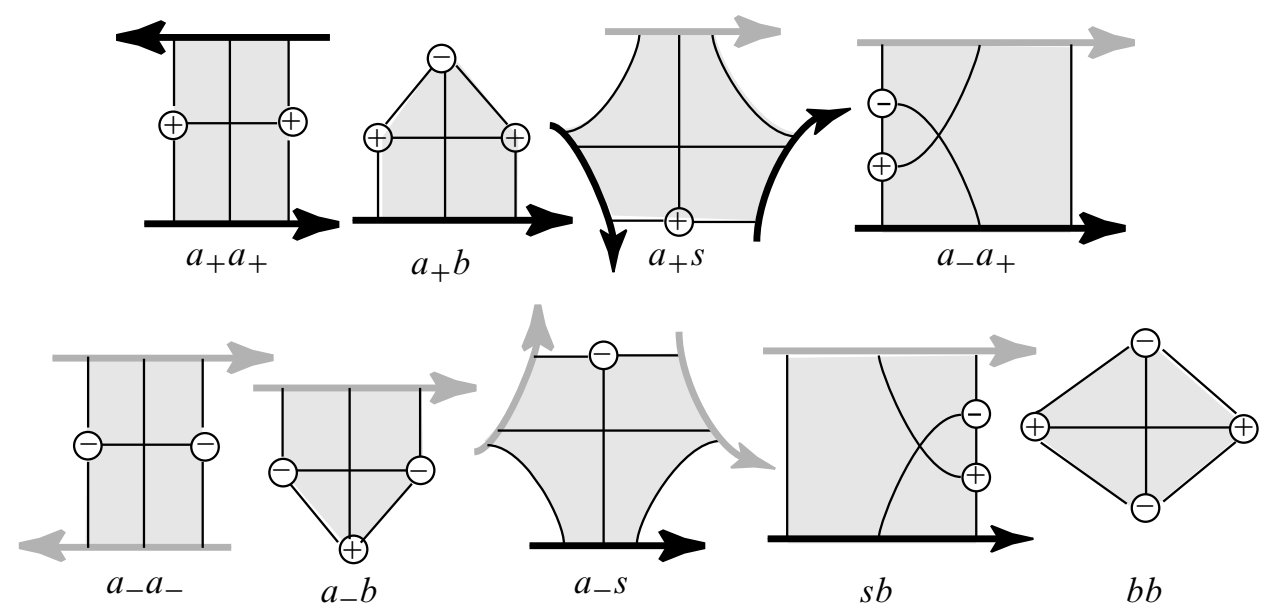

Figure 7: Possible tile types in the foliation of $\mathcal{C A}$. The black (resp. gray) arcs represent subarcs of $X_{+}$(resp. $X_{-}$).

The foliation determines a cellular decomposition of $\mathcal{P A}$ which goes over to a cellular decomposition of $S^{2}$ on shrinking the 2 boundary components to points. Letting $V, E$ and $F$ be the number of vertices, edges and faces, the fact that $\chi\left(S^{2}\right)=2$ shows that on each component of the foliated surface $\mathcal{P} A$ we have $V+2-E+F=2$. Each tile has four edges and each edge is an edge of exactly 2 tiles, so that $E=2 F$. Combining this with the previous equation we learn that $V=F$. Let $v_{\epsilon}$ (resp. $s_{\epsilon}$ ) be the number of vertices (resp. singularities) of sign $\epsilon$ in $\mathcal{P A}$. Since there is exactly one singularity of either sign in each tile, it follows that

$$
\left(v_{+}+v_{-}\right)=\left(s_{+}+s_{-}\right) .
$$

We return to the situation where $X_{+}$and $X_{-}$are transversal knots $T X_{+}, T X_{-}$, also $\mathcal{T A}$ is a transversal clasp annulus, so that the isotopy across $\mathcal{T A}$ is a transversal 
isotopy. We claim that the signed vertices and singularities also detect the difference between the Bennequin invariants $\beta\left(T X_{+}\right)$and $\beta\left(T X_{-}\right)$. For, recall that the isotopy was constructed from the transversal Markov tower of (2). The moves that relate adjacent braids in that tower are braid isotopy and positive stabilizations and destabilizations. While braid isotopy may change the braid foliation, it does not change the numbers $v_{+}, v_{-}, s_{+}, s_{-}$, so the only issue is what happens during stabilizations and destabilizations. Let's consider a positive destabilization. We have a single trivial loop around the $z$ axis, with a positive crossing. We have $d \theta>0$ along the entire length of the loop since we are working with a closed braid. For a positive crossing we have $d z \geq 0$ throughout the loop as well. Therefore the inequality $d z / d \theta>-\rho^{2}$ is true for all non-zero real values of $\rho$. Crossing the $z$-axis to destabilize the braid results in at least one singular point, where $d \theta=0$, but if we continue to keep $d z \geq 0$ then in the limit, as $-\rho^{2} \rightarrow 0$ from the negative real numbers, $d z / d \theta$ goes to $\infty$ through the positives. Therefore $d z / d \theta \neq-\rho^{2}$ at any stage in the isotopy. Therefore the isotopy is transversal. After the isotopy the number of positive vertices will have decreased by 1 and the number of positive singularities also will have decreased by 1 so that the difference between them is zero. Now recall that the Bennequin invariant $\beta(T X)$ of a transversal knot is the difference between its braid index and its algebraic crossing number. Thus the Bennequin invariant is preserved during a positive destabilization. The situation is the same for a positive stabilization. In particular, if we study the sequence of moves that take us from $T X_{+}$to $T X_{-}$in (2) we see that:

$$
\beta\left(X_{+}\right)-\beta\left(X_{-}\right)=\left(s_{+}-s_{-}\right)-\left(v_{+}-v_{-}\right)=0 .
$$

Using Equations (3) and (4), it follows that if $\mathcal{T A}$ is swept out during a transversal isotopy, then:

$$
v_{+}=s_{+} \text {and } v_{-}=s_{-} .
$$

We now wish to apply our knowledge about transversal isotopies to a specific situation. Let $X \in \mathcal{X}$ be a braid in braid structure $(\mathbf{H}, \mathbf{A})$ such that $X \in \mathcal{B}_{\min }(\mathcal{X})$. Let $T \Delta \subset \mathbb{R}^{3}$ be an embedded disc, with boundary $\partial(T \Delta)$ the union of two intervals $a_{1} \cup a_{2}$, where:

(1) $a_{1} \subset X$.

(2) $\left(X \backslash a_{1}\right) \cup a_{2}=X^{\prime} \in \mathcal{B}(\mathcal{X})$. In particular, $X \cap i n t(T \Delta)=\varnothing$ and $X^{\prime}$ is isotopic to $X$ by the motion of $a_{2}$ to $a_{1}$ across $T \Delta$.

(3) The positioning of $T \Delta$ in $(\mathbf{H}, \mathbf{A})$ is such that the braid foliation of $T \Delta$ is either by parallel arcs transverse to the boundary, or is the union of tiles from Figure 7 with the exception that we allow the foliation of $T \Delta$ to have two cusp points near the points $a_{1} \cap a_{2}$. 
(4) Orienting $T \Delta$ so as to be consistent with the orientation of $a_{2}$, the tiling of $T \Delta$ is such that $\left(v_{+}-v_{-}\right)-\left(s_{+}-s_{-}\right)=0$. Thus, the graph $X \cup a_{2}$ is in braid position and its edges have natural orientations.

We call such a disc $T \Delta$ a transversal disc between $X$ and $X^{\prime}$.

Before we can state our next lemma, we need one more concept about braid foliations. It is to be expected that the union of the singular leaves will contain most of the information about the braid foliation, but in our situation there is additional information. The parity information associated with the vertices and singularities of a braid foliation allows us to define four graphs $G_{+,+}, G_{+,-}, G_{-,+}$and $G_{-,-}$. Let $G_{\epsilon, \delta}$, where $\epsilon$ and $\delta$ are \pm , be the graph that contains the

\begin{tabular}{|c|c|c|c|}
\hline$G_{+,+}$ & $G_{-,-}$ & $G_{+,-}$ & $G_{-,+}$ \\
\hline &
\end{tabular}

Figure 8: The graph $G_{\epsilon, \delta}$ includes all singular leaves through vertices of sign $\epsilon$ and singularities of sign $\delta$. The thick edges illustrate the passage of $G_{+,+}, G_{-,-}, G_{+,-}, G_{-,+}$through a $b b-$ tile.

vertices of sign $\epsilon$ and connects them together using edges that are contained in singular leaves of sign $\delta$. By definition $G_{\epsilon, \delta} \cap G_{-\epsilon,-\delta}=\varnothing$. See Figure 8, which illustrates how the 4 graphs intersect a $b b$-tile. The reader should not have any difficulty in seeing how the 4 graphs pass through the tiles in Figure 7. Observe that for all the other tiles there will be vertex endpoints of the graphs on the boundary, because the singular leaves have endpoints on the boundary.

Lemma 3 Let $T \Delta$ be a transversal disc between the braids $X$ and $X^{\prime}$. Then, when $X$ and $X^{\prime}$ are viewed as transversal knots in the standard contact structure, the isotopy across $T \Delta$ is a transversal isotopy. In particular, if $\mathcal{C} \subset G_{+,+}$(resp. $G_{-,-}$) is a component of the graph in $T \Delta$ then $\mathcal{C}$ is a tree having a single endpoint on $a_{1}$ (resp. $\left.a_{2}\right)$. 
Proof From [7] we know that a positive stabilization or destabilization corresponds to a transversal isotopy. From Section 3.4 of [6] we know if $\delta \subset T \Delta$ is a regular neighborhood of a single edge of $G_{-,-}$which has an endpoint on $a_{2}$ then the isotopy of $a_{2}$ across $\delta$ corresponds to a positive stabilization of $a_{2}$. Similarly, if $v \subset G_{+,+}$ is an endpoint of a component and, also, a valence one vertex in the foliation of $T \Delta$ then from Section 3.3 of [6] we know we can eliminate $v$ by a positive destabilization. Thus, if every component of $G_{-,-}$is a tree having a single endpoint on $a_{2}$ we can eliminate the graph $G_{-,-}$through a sequence of positive stabilizations of $a_{2}$. All that will remain is the graph $G_{+,+}$. If the components of $G_{+,+}$are trees each having an endpoint on $a_{1}$ then through a sequence of positive destabilizations (starting at vertex endpoints) we can eliminate the components of $G_{+,+}$. What will remain of $T \Delta$ will be trivially foliated and an isotopy across a trivially foliated transverse tab corresponds to a braid isotopy in the complement of $\mathbf{A}$. But, such braid isotopies are transversal isotopies. Thus, to establish the main assertion of the lemma we need to establish the assertions that the components of our graphs $G_{ \pm, \pm}$are trees having endpoints on the appropriate $a_{i}$ arc of $T \Delta$.

The initial fact that each component of $G_{ \pm, \pm}$must be a tree follows from Theorem 3.1 of [3]. We also learn from Theorem 3.1 of [3] that if $\mathcal{C} \subset G_{+,+}$has more than one endpoint on $a_{1}$ then after a sequence of braid isotopies (which are in the form of change of fibration) and exchange moves, we can destabilize $a_{1}$. But this would imply that $X$ was not of minimal braid index. So any component of $G_{+,+}$can have at most one endpoint on $a_{1}$. Now notice that for those components of $G_{+,+}$having a single endpoint on $a_{1}$ the number of positive vertices equals the number of singularities And, for those that don't have an endpoint on $a_{1}$ the number of positive vertices is always one more than the number of singularities. But, since $v_{+}=s_{+}$we have to have all components of $G_{+,+}$having an endpoint on $a_{1}$. Finally, if $\mathcal{C} \subset G_{-,-}$is a component that has more than one endpoint on $a_{2}$ then there is a path in $\mathcal{C}$ that splits off a subdisc of $T \Delta$ that contains a component of $\mathcal{C}^{\prime} \subset G_{+,+}$. But, then $\mathcal{C}^{\prime}$ could not have an endpoint on $a_{1}$, which contradicts what we just proved.

We need a few more definitions before we can state our main application of Lemma 3, ie, Proposition 2 below. We say that a clasp $\operatorname{arc} \gamma \subset \mathcal{T A}$ is short for $X_{+}$(resp. $X_{-}$) if there exists a triangular disc $\Delta_{+} \subset \mathcal{P} \mathcal{A}$ (resp. $\Delta_{-} \subset \mathcal{P A}$ ) such that $a_{1} \cup a_{2} \cup a_{3}=\partial \Delta_{+}$ (resp. $=\partial \Delta_{-}$) where $a_{1}$ in contained in an $a_{+}-\operatorname{arc}$ (resp. $\left.a_{-}-\operatorname{arc}\right) ; a_{2}=\gamma_{+}$(resp. $\left.=\gamma_{-}\right) ; a_{3} \subset e^{-1}\left(X_{+}\right)$(resp. $e^{-1}\left(X_{-}\right)$); and, $\Delta_{+}$(resp. $\left.\Delta_{-}\right)$is trivially foliated.

We say that a clasp arc $\gamma_{-}$(resp. $\left.\gamma_{+}\right)$is parallel to a component $\mathcal{C}$ of $G_{+,+}$(resp. $\left.G_{-,-}\right)$. if there exists an edgepath $\mathcal{E} \subset \mathcal{C}$ and a rectangular disc $R \subset \mathcal{P A}$ such that;

(1) $\partial R=a_{1} \cup a_{2} \cup a_{3} \cup a_{4}$, where $a_{1}=\gamma_{-}$(resp. $\left.a_{1}=\gamma_{+}\right), a_{2}$ is a subarc contained in a leaf of the foliation, $a_{3} \subset X_{-}$(resp. $a_{3} \subset X_{+}$) and $a_{4}=\mathcal{E}$. 
(2) $\operatorname{int}(R)$ does not contain any vertices or singularities of the foliation.

(A foliated neighborhood of the edgepath $\mathcal{E}$ which contains $\gamma_{-}$(or $\gamma_{+}$) and only the vertices and singularities in $\mathcal{E}$ will play the part that tab neighborhoods of clasp arcs played in [6].)

Proposition 2 Let $\mathcal{T} \mathcal{X}$ be a transversal oriented knot type and $\left(T X_{+}, T X_{-}\right)$be a pair of transversal closed braids which represent $\mathcal{T} \mathcal{X}$. Assume that the braid index $b\left(T X_{+}\right)=b\left(T X_{-}\right)=m$, where $m$ is minimal for all closed braid representatives of the topological knot type $\mathcal{X}$. Then the transversal clasp annulus $\mathcal{T}$ A associated with ( $\left.T X_{+}, T X_{-}\right)$supports a braid foliation, which satisfies the following conditions:

(a) The leaves of the the braid foliation are type $s-, a_{+}-, a_{-}-$or $b-\operatorname{arcs}$ and the singularities correspond to those listed in Figure 7.

(b) The components of $G_{+,+}$(resp. $\left.G_{-,-}\right)$are trees, having a single endpoint on $T X_{-}\left(\operatorname{resp} . T X_{+}\right)$.

(c) For each clasp arc $\gamma^{i} \subset \mathcal{T A}$ at least one of its associated pre-images $\gamma_{+}^{i}$ or $\gamma_{-}^{i} \subset \mathcal{P A}$ is either short or is parallel to an edgepath in the pre-image of $G_{+,+}$ or $G_{-,-}$.

Proof Our proof will use Lemma 3. Let $\mathcal{T A}$ be a transversal clasp annulus associated with the pair $\left(T X_{+}, T X_{-}\right)$. Inside the pre-image $\mathcal{P A}$ we have the pre-image of the clasp $\operatorname{arcs}\left\{\gamma_{+}^{1}, \gamma_{-}^{1}, \cdots, \gamma_{+}^{\mathrm{k}}, \gamma_{-}^{\mathrm{k}}\right\}$ and their extension $\operatorname{arcs}\left\{g_{+}^{1}, g_{-}^{1}, \cdots, g_{+}^{\mathrm{k}}, g_{-}^{\mathrm{k}}\right\}$. Recall that we have the graph

$$
\mathcal{G}=T X_{+} \cup T X_{-} \cup\left(\cup_{1 \leq i \leq \mathrm{k}} \gamma^{i}\right) \cup\left(\cup_{1 \leq i \leq \mathrm{k}} e\left(g_{+}^{i}\right)\right) \cup\left(\cup_{1 \leq i \leq \mathrm{k}} e\left(g_{-}^{i}\right)\right),
$$

and that it is both in braid position and transverse to the standard contact structure. By a small isotopy near the vertices of this graph we can assume that it is differentiable. Thus, in $\mathcal{P A}$ each edgepath $g_{ \pm}^{i} \cup \gamma_{ \pm}^{i}$ will split off a subdisc of $\mathcal{P A}$ that has two cusps.

We now use the fact that the edgepaths $\left\{\left(g_{+}^{1} \cup \gamma_{+}^{1}\right),\left(g_{-}^{1} \cup \gamma_{-}^{1}\right), \cdots,\left(g_{+}^{\mathrm{k}} \cup \gamma_{+}^{\mathrm{k}}\right),\left(g_{-}^{\mathrm{k}} \cup\right.\right.$ $\left.\left.\gamma_{-}^{\mathrm{k}}\right)\right\} \subset \mathcal{P} \mathcal{A}$ are staggered. Let $\Lambda=\left\{s^{1}, \cdots, s^{1}\right\} \subset \mathcal{P A}$ be a set of leaves in the pre-image of the characteristic foliation of $\mathcal{T A}$ such that:

(i) Each component of $\mathcal{P} \mathcal{A} \backslash \Lambda$ is a subdisc that contains at most one $g_{ \pm}^{i} \cup \gamma_{ \pm}^{i}$ edgepath. (We allow for the possibility that a component contains no such edgepath.) 


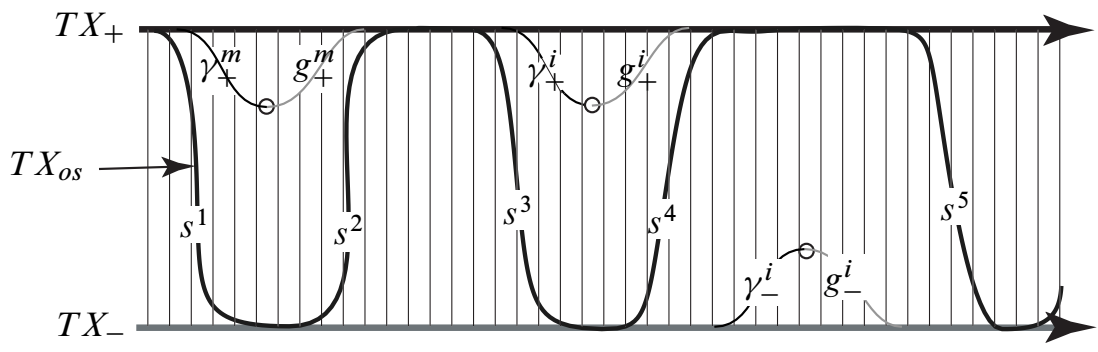

Figure 9: The graph $\mathcal{G}$, as it appears in the annulus $\mathcal{P A}$.

(ii) By a small isotopy, the components of $\Lambda \subset \mathcal{P A}$ can be made transverse to the characteristic foliation so that the graph $T X_{+} \cup T X_{-} \cup e(\Lambda)$ is: differentiable and transverse in the standard contact structure; and, there is a smooth loop $T X_{o s} \subset T X_{+} \cup T X_{-} \cup e(\Lambda)$ that oscillates away from the clasps; ie, there exists arcs $\left\{x_{+}^{1}, \cdots, x_{+}^{1}\right\} \subset T X_{+}$and $\left\{x_{-}^{1}, \cdots, x_{-}^{1}\right\} \subset T X_{-}$such that, when the leaves of $e(\Lambda)$ are positioned transversal in $\mathcal{T A}$ the smooth loop $T X_{o s}=$ $x_{+}^{1} \cup s^{1} \cup x_{-}^{1} \cup s^{2} \cup x_{+}^{2} \cup s^{3} \cup x_{-}^{3} \cup \cdots \cup s^{1}$ does not intersect any clasp arcs.

Notice that $T X_{o s} \subset \mathcal{T A}$ is transversally isotopic to $T X_{+}$, to $T X_{0}$ and to $T X_{-}$.

We now consider the expanded graph

$$
\Gamma=T X_{+} \cup T X_{-} \cup T X_{o s} \cup\left(\cup_{1 \leq i \leq \mathrm{k}} \gamma^{i}\right) \cup\left(\cup_{1 \leq i \leq \mathrm{k}} e\left(g_{+}^{i}\right)\right) \cup\left(\cup_{1 \leq i \leq \mathrm{k}} e\left(g_{-}^{i}\right)\right) \subset \mathcal{T} \mathcal{A}
$$

The graph $\Gamma$ is a transversal graph in the standard contact structure. Except for the portion of it that corresponds to $e(\Lambda)$, it is also a braided graph. We now make it entirely braided by applying first the Bennequin trick to $e(\Lambda)$ then extending this transversal isotopy of $\Gamma$ to all of $\left(\mathbb{R}^{3}, \xi\right)$ by Eliashberg's Lemma (Lemma 2). The resulting $\mathcal{T A}$ will still be a transversal clasp annulus with $T X_{o s}$ now being in braid position.

We claim that the braid foliation on $\mathcal{T A}$ must be a tiling. If it is not then there will exist a leaf in the braid foliation of $c \subset \mathcal{T A}$ that is a circle. But, notice that, due to the fact the $\Gamma$ is in braid position, its edges can be consistently oriented. Thus, $c \cap \Gamma=\varnothing$ due to orientation considerations. We then have $c \subset \mathcal{T A} \backslash \Gamma$ and $c$ will therefore bound an embedded disc $\Delta_{c} \subset \mathcal{T A}$. Since $c \subset H_{\theta}$ for some $H_{\theta} \in \mathbf{H}$ we see that it also bounds a $\Delta_{\theta} \subset H_{\theta}$ which, without loss of generality, we can assume is inner most in $H_{\theta}$. After smoothing the corners around $c$ we will have a smooth 2-sphere $\Delta_{c} \cup_{c} \Delta_{\theta}$ whose characteristic foliation will be radial in $\Delta_{\theta}\left(\subset \Delta_{c} \cup_{c} \Delta_{\theta} \cong S^{2}\right)$ with one elliptic point. So the characteristic foliation on $\Delta_{c}$ must also have an elliptic point. 
But, $\Delta_{c} \subset \mathcal{T A}$ and the characteristic foliation on $\mathcal{T A}$ is trivial. We conclude that the circle leaf $c$ could not have existed in the braid foliation of $\mathcal{T A}$. Thus $\mathcal{T A}$ is tiled.

Next we establish that each clasp $\operatorname{arc} \gamma^{i}$ is associated to the preimage of either a short clasp arc or a parallel clasp arc in $\mathcal{P} \mathcal{A}$. So let $\mathcal{E}_{-}^{i}=\gamma_{-}^{i} \cup g_{-}^{i} \subset \mathcal{P} \mathcal{A}$ be a clasp arc and its extension. Notice that $e\left(\mathcal{E}_{-}^{i}\right) \subset \mathcal{T A}$ splits off an embedded disc, $T \Delta_{-}^{i} \subset \mathcal{T A}$, that has an induced trivial characteristic foliation. So there is a transversal isotopy of $e\left(\mathcal{E}_{-}^{i}\right)$ across $T \Delta_{-}^{i}$ into $T X_{-}$. Our initial goal is to establish that $T \Delta_{-}^{i}$ is a transversal disc. But, this is clear because when we consider the braid foliation of $\mathcal{T A}$ restricted to $T \Delta_{-}^{i}$ we see that it has an induced tiling and that one of its boundary arcs is on a braid of minimal index. Moreover, since $T \Delta_{-}^{i}$ illustrates a transversal isotopy, for the tiling of $T \Delta_{-}^{i}$ we have the equation $\left(v_{+}-v_{-}\right)-\left(s_{+}-s_{-}\right)=0$.

If the induced braid foliation on $T \Delta_{-}^{i}$ is trivial, then $\gamma_{-}^{i}$ is short. So assume the braid foliation on $T \Delta_{-}^{i}$ is non-trivial. Then we apply Lemma 3 and conclude that every component of $G_{\epsilon, \epsilon}, \epsilon= \pm$, is a tree, also each component of $G_{-,-}$will have one endpoint on $e\left(\mathcal{E}_{-}^{i}\right)$, and each component of $G_{+,+}$will have one endpoint on $T X_{-}$. We can assume that the endpoints of $G_{-,-}$on $e\left(\mathcal{E}_{-}^{i}\right)$ are away from the point $e\left(\gamma_{-}^{i} \cap g_{-}^{i}\right) \subset T X_{+}$which cannot be moved. Now, by stabilizing positively $e\left(\mathcal{E}_{-}^{i}\right)$ along the components of $G_{-,-}$in $T \Delta_{-}^{i}$ we can force the elimination of $G_{-,-}$in the resulting transversal disc, which by abuse of notation we still call $T \Delta_{-}^{i}$. We now eliminate the endpoint vertices of any component of $G_{+,+}$in $T \Delta_{+,+}$via positive destabilization, ie, we eliminate valence one vertices of $G_{+,+}$in $T \Delta_{-}^{i}$. (See Sections 3.3 and 4.3 of [6].) This can be done so long as the neighborhood of the valence one vertex does not contain the point $e\left(\gamma_{-}^{i} \cap g_{-}^{i}\right)$. After the elimination of all possible valence one vertices we will have the resulting $T \Delta_{-}^{i}$ containing only a linear component of $G_{+,+}$and $\gamma_{-}^{i}$ will, thus, be parallel to an edgepath in $G_{+,+}$.

Finally, we notice that $T X_{o s} \subset \mathcal{T A}$ decomposes $\mathcal{T A}$ into a union of transversal discs. The reasoning is similar to that of why $T \Delta_{-}^{i}$ was a transversal disc. Namely, any disc component $T \Delta \subset \mathcal{T A} \backslash T X_{o S}$ is an embedded disc having a trivial characteristic foliation coming from the characteristic foliation of $\mathcal{T A}$. Thus, an isotopy of $T X_{o s}$ across $T \Delta$ is a transversal isotopy. This implies that for the braid foliation on $\mathcal{T A}$ restricted to $T \Delta$ we again have the equation $\left(v_{+}-v_{-}\right)-\left(s_{+}-s_{-}\right)=0$. Applying Lemma 3 to $T \Delta$ we see that, since one boundary arc of $\partial T \Delta$ is on a braid of minimal index, the components of $G_{\epsilon, \epsilon}$ are trees having endpoints on the appropriate subarcs of the boundary of $T \Delta$. This implies that the components of $G_{\epsilon, \epsilon} \subset \mathcal{T A}$ are trees having single endpoints on $T X_{-\epsilon}$. Our proof is complete.

\subsection{A weak transversal MTWS}

We are now ready to state and prove our transverse version of the MTWS. 
By Proposition 1 we know that there is a transversal clasp annulus $\mathcal{T A}$ associated to any pair $\left(T X_{+}, T X_{-}\right)$. The characteristic foliation on $\mathcal{T A}$, which is trivial, illustrates that the isotopy of $T X_{+}$to $T X_{-}$through $\mathcal{T A}$ is in fact a transversal isotopy. We also have a second foliation on $\mathcal{T A}$, the singular braid foliation that is induced by the intersection of $\mathcal{T A}$ with the half-planes of the standard braid fibration $\mathbf{H}$ of $\mathbb{R}^{3}$. In [6] this braid foliation was used to construct templates $\left(\mathcal{D}_{+}, \mathcal{D}_{-}\right)$of the MTWS. Specifically, using the graphs $G_{\epsilon, \delta}$ coming from the braid foliation, stabilizations and destabilizations along singular leaves in the complement of clasp arcs went to make up the isotopies used in the sequences taking $\mathcal{D}_{+}$to $\mathcal{D}_{-}$. However, our main concern, in [6], was in controlling the change in braid index in the passage from $\mathcal{D}_{+}$to $\mathcal{D}_{-}$.

Our concern here is somewhat different. Suppose we have a template $\left(\mathcal{D}_{+}, \mathcal{D}_{-}\right)$such that, when $T X_{+}$and $T X_{-}$are viewed as braids, we see that $\mathcal{D}_{+}$carries $T X_{+}$and $\mathcal{D}_{-}$carries $T X_{-}$via a common braiding assignment to the blocks of our template. We ask whether there is a Markov tower which takes $\mathcal{D}_{+}$to $\mathcal{D}_{-}$using only positive stabilizations/destabilizations of braid isotopies? In other words, by equation (2), is there a transversal isotopy from $\mathcal{D}_{+}$to $\mathcal{D}_{-}$? Having the templates in hand, we are willing to decompose the isotopy into the moves in a markov tower. Our only concern is whether that tower can be realized by transversal isotopies.

Recall the notation $\mathcal{T}(m, n)$ for the set of all templates in Theorem 1. A template will be said to be a transversal template if there is a sequence of positive stabilizations, positive destabilizations and braid isotopies that take $\mathcal{D}_{+}$to $\mathcal{D}_{-}$. Our weak transversal MTWS is:

Theorem 2 (A weak transversal MTWS) Let $\mathcal{T} \mathcal{X}$ be a transversal oriented knot type and let $\left(T X_{+}, T X_{-}\right)$be a pair of transversal closed braids which represent $\mathcal{T} \mathcal{X}$. Assume that the braid index $b\left(T X_{+}\right)=b\left(T X_{-}\right)=m$, where $m$ is minimal for all closed braid representatives of the topological knot type $\mathcal{X}$. Then there exist $T X_{+}^{\prime}$ and $T X_{-}^{\prime} \in \mathcal{B}_{\min }(\mathcal{X})$, and a transversal template $\left(\mathcal{D}_{+}, \mathcal{D}_{-}\right) \in \mathcal{T}(m, m)$ such that:

(1) $T X_{+}$(resp. $\left.T X_{-}\right)$is exchange equivalent to $T X_{+}^{\prime}$ (resp. $T X_{-}^{\prime}$ ). In particular, $T X_{+}\left(\right.$resp. $\left.T X_{-}\right)$is transversally isotopic to $T X_{+}^{\prime}\left(\operatorname{resp} . T X_{-}^{\prime}\right)$.

(2) $T X_{+}^{\prime}$ is carried by $\mathcal{D}_{+}$and $T X_{-}^{\prime}$ is carried by $\mathcal{D}_{-}$, via a common braiding assignment to $\mathcal{D}_{+}$and $\mathcal{D}_{-}$.

The reader who is interested mainly in the applications to contact topology may now wish to skip ahead to the applications in Section 3 in this paper. On the other hand, the reader who has studied [6] should have little difficulty in following the proof of Theorem 2, below. 
Proof of Theorem 2 Our proof will depend on the proof in [6] of Theorem 1, stated above. We will pinpoint the precise sections of [6] which are needed as they are used in our proof of Theorem 2 by giving section numbers and section headings (which we highlight with italics) from [6].

We are given the transversal closed braids $\left(T X_{+}, T X_{-}\right)$which represent the transversal knot type $\mathcal{T} \mathcal{X}$. By the construction in Proposition 1 we know that there is an associated transversal clasp annulus for $\left(T X_{+}, T X_{-}\right)$. By Proposition 2 we know that such a transversal clasp annulus will have a braid foliation such that: the components of $G_{+,+}$ and $G_{-,-}$will be trees each having a single endpoint on the appropriate boundary curve of $\mathcal{T A}$; and each clasp arc will either be short, or parallel to a component of $G_{+,+}$or $G_{-,-}$. This is equivalent, in the language of [6], to saying that the clasp arcs have been placed in tab neighborhoods. See Section 4.3 of [6], Construction of the tabs.

We ask whether the constructions in Sections 4.4, The two finger moves, and 4.5, Creating symmetric normal neighborhoods of the clasp arcs, of [6] can be used to produce symmetric normal neighborhoods for our transverse clasp arcs? It is easy to check that the finger moves preserve the fact that our graphs are trees. Another way to see the same thing is to observe that all the alterations to the braid foliation of $\mathcal{T A}$ which are used in Section 4.5 of [6] preserve the Bennequin number equality $\left(v_{+}-v_{-}\right)-\left(s_{+}-s_{-}\right)=0$. Thus the clasp arcs in our transversal clasp annulus can be assumed to have symmetric normal neighborhoods, as established in Section 4.5 of [6].

Moving on, Section 5 of [6] describes (in the topological setting) the ways in which one pushes $X_{+}$across $\mathcal{C A}$ to $X_{-}$. These are the tools that are used to construct the templates of [6]. We can of course, drop the restrictions to transverse knots and consider our $T X_{+}$and $T X_{-}$as $X_{+}$and $X_{-}$, and follow the moves used in [6] to push across $\mathcal{C A}$. The question we need to address is whether those moves can be realized by transversal isotopy?

Clearly the complexity function of Section 5.1 of [6] is equally valid with or without the extra structure provided by contact topology.

We turn our attention to Section 5.2, Pushing across $\mathcal{C A}$ with exchange moves and destabilizations. The alterations that are used in the proof of Proposition 5.2.1 are (i) destabilizations of $X_{+}$, (ii) exchange moves and (iii) changes in foliation. However, destabilizations of $X_{+}$cannot occur in our situation because of our assumption that the topological braid index of $T X_{+}$is minimal. It was proved in [7] that exchange moves can always be realized by transversal isotopy. Note that exchange moves on $T X_{+}$ (resp. $T X_{-}$) do not change the fact that $G_{+,+}$(resp. $G_{-,-}$) is a union of trees. As for changes in foliation, they are realized by braid isotopy. By Lemmas 1 and 2 of this 
paper, the required braid isotopies can be realized by transversal isotopies. So we can assume that we are in the situation at the end of Section 5.2 of [6]. Putting this another way (see Section 6.1 of [6]) we can assume that we have replaced the original pair of closed braids $\left(T X_{+}, T X_{-}\right)$by the new pair of transversal closed braids $\left(T X_{+}^{\prime}, T X_{-}^{\prime}\right)$ of assertion (1) of Theorem 2 of this paper.

We move on to Section 5.3 and Section 5.4, where microflypes and flypes are used to shorten the clasp arcs and push across $\mathcal{C A}$. By the construction in [6] all of the flypes can be decomposed into stabilizations, microflypes, and destabilizations. The parity for the microflypes is totally dependent on whether the clasp arcs are parallel to one of the graphs $G_{\epsilon, \epsilon}$ on the one hand, or one of the graphs $G_{\epsilon,-\epsilon}$ on the other hand. Since all of our clasp arcs are either short or parallel to $G_{+,+}$or $G_{-,-}$, it follows that in the braid foliation of our $\mathcal{T A}^{\prime}$ any microflype across a thin annulus will be realizable by a transversal isotopy. When the microflypes are reconstituted into a larger flype, the amalgamating conditions insure that the signs are consistent, showing that the resulting flypes are all transverse. The same is true in Section 5.6 of [6], Pushing across a region with a $G$-flype foliation, where we study the amalgamation of flypes into $\mathrm{G}-$ flypes. The resulting G-flypes will all be transversal because our graphs $G_{+,+}$and $G_{-,-}$have the needed sign data for transversal isotopy, and because without consistent signs there can be no amalgamation of flypes into G-flypes.

We pass to G-exchange moves, studied in Section 5.7. While G-exchange moves are nothing more than inter-related sequences of exchange moves, it seems possible that the 'looping' of strands which is needed for the G-exchange moves (illustrated nicely in the example of Figure 10 of [6]) might not be transversal. However, when we look more carefully at G-exchange moves, we see that they can always be replaced by sequences of stabilizations, braid isotopies and destabilizations (see Figure 5 of [6]), and the signs of the stabilizations and destabilizations can be chosen to be either positive or negative for exchange moves, so again (at the expense of giving up the global nature of the moves, which are not the issue for us here), we have transversal isotopies.

Finally, for a motion across a standard annulus, discussed in Section 5.8 of [6], Pushing across a standard annulus, we need to consider whether the components of $G_{+,+}$and $G_{-,-}$are homotopic to $S^{1}$ or to the unit interval. If the former, then the motion across a standard annulus will always require a negative stabilization and destabilization. If they are homotopic to an interval, the motion will utilize one positive stabilization, some number of exchange moves, and one positive destabilization. But since $\mathcal{T} \mathcal{A}^{\prime}$ satisfies the conclusions of Proposition 2, the components of $G_{+,+}$and $G_{-,-}$will be homotopic to intervals and the motion will correspond to a transversal isotopy.

The recipe of Section 5.5 of [6], Constructing the template $\left(\mathcal{D}_{+}, \mathcal{D}_{-}\right)$, now tells us how to construct a template $\left(\mathcal{D}_{+}, \mathcal{D}_{-}\right)$that carries the pair $\left(T X_{+}^{\prime}, T X_{-}^{\prime}\right)$. We have proved 
that $\left(\mathcal{D}_{+}, \mathcal{D}_{-}\right)$is in fact a transversal clasp annulus, because we proved that every move that is needed can be realized by a transversal isotopy. The proof of Theorem 2 is complete.

Remark The main way in which our tranversal MTWS is restrictive and therefore 'weak' is as follows. In [6] we required $X_{-}$to have minimal braid index, but here we require both $T X_{+}$and $T X_{-}$to have minimal topological braid index. Notice that this places a very severe restriction on the transversal knot types which are covered, because in the generic case one expects that only very special transversal knot types, for example those which have maximal Bennequin invariant, will be represented by closed braids with minimum topological braid index.

\section{Transversal simplicity and its failure}

In this section we develop our examples illustrating the failure of transversal simplicity. In particular, we will produce examples of pairs of closed braids, $\left(X_{+}, X_{-}\right)$such that: $X_{+}, X_{-} \in \mathcal{B}_{\min }(\mathcal{X})$; their associated transversal knots $T X_{+}$and $T X_{-}$will have $\beta\left(T X_{+}\right)=\beta\left(T X_{-}\right)$; but, $T X_{+}$is not transversally isotopic to $T X_{-}$. Our over-riding strategy will be to employ Theorem 1 and Theorem 2 through the following line of reasoning.

Suppose that $T X_{+}$and $T X_{-}$were transversally isotopic. Then by Theorem 2, there must be a transversal template $\left(\mathcal{D}_{+}, \mathcal{D}_{-}\right)$that carries the pair $\left(X_{+}, X_{-}\right)$. By Theorem 1 we know that there will only be finitely many possible templates, and we will have judiciously chosen $\left(X_{+}, X_{-}\right)$so that $\left(\mathcal{D}_{+}, \mathcal{D}_{-}\right)$is the unique template of $\mathcal{T}(m, m)$ that carries $\left(X_{+}, X_{-}\right)$. We will then demonstrate that $\left(\mathcal{D}_{+}, \mathcal{D}_{-}\right)$also carries links $\left(Y_{+}, Y_{-}\right)$for which component-wise the Bennequin invariant is not preserved. This will establish that $\left(\mathcal{D}_{+}, \mathcal{D}_{-}\right)$could not have been a transversal template.

At the end of the section we pose some open problems.

\subsection{The failure of transversal simplicity for certain closed 3-braids}

Theorem 3 There exist infinitely many transversal knot types of braid index 3 which are not transversally simple. In particular, consider the collection of infinitely many pairs of transversal knots $\left(T X_{+}, T X_{-}\right)$defined by the pairs of transversal closed 3-braids

$$
T X_{+}=\sigma_{1}^{2 p+1} \sigma_{2}^{2 r} \sigma_{1}^{2 q} \sigma_{2}^{-1}, \quad T X_{-}=\sigma_{1}^{2 p+1} \sigma_{2}^{-1} \sigma_{1}^{2 q} \sigma_{2}^{2 r}
$$


where $p+1 \neq q \neq r$ and $p, q, r>1$ Then, the transversal knot types $\mathcal{T} \mathcal{X}_{+}$and $\mathcal{T} \mathcal{X}_{-}$ associated to each pair belong to the same topological knot type and have the same $\beta$-invariant, but they do not represent the same transversal knot type.

Proof of Theorem 3 To begin we verify that $\left[\mathcal{T} \mathcal{X}_{+}\right]_{\text {top }}=\left[\mathcal{T} \mathcal{X}_{-}\right]_{\text {top }}$ and $\beta\left(T X_{+}\right)=$ $\beta\left(T X_{-}\right)$. The closed braids $T X_{+}$and $T X_{-}$of Theorem 3 are carried by the negative flype template (see Figure 4), with the strands all assigned weight 1 . The braiding assignments to the blocks are $\sigma_{1}^{2 p+1}, \sigma_{2}^{2 r}$ and $\sigma_{1}^{2 q}$ to $P, R$ and $Q$ respectively. There is a template isotopy from the left diagram to the right diagram which preserve knot or link type for any braiding assignment to the blocks, so $\left[T X_{+}\right]_{\text {top }}=\left[T X_{-}\right]_{\text {top }}$. If a transversal knot is defined by a closed braid $T X$, then its $\beta$-invariant is given by the difference between the algebraic crossing number of the diagram and the braid index, therefore $\beta\left(T X_{+}\right)=\beta\left(T X_{-}\right)=2 p+2 r+2 q-3$, as claimed.

Let us assume that there is a transversal isotopy from the transverse closed braid $T X_{+}$ to the transverse closed braid $T X_{-}$. Theorem 2 would imply that there is a transversal 3 -braid template that carries the braid $\left[T X_{+}\right]_{\text {top }}$ and the braid $\left[T X_{-}\right]_{\text {top }}$. However, we know that every transverse isotopy is also a topological isotopy, and we know a great deal about knots that are defined by closed 3-braids, so we can place restrictions on the topological isotopy. Let $\mathcal{X}=\left[T X_{+}\right]_{\text {top }}=\left[T X_{-}\right]_{\text {top }}$ be the topological knot type that is defined by the transverse closed braids $T X_{+}, T X_{-}$of Theorem 3. By the main theorem in [4], a knot which is determined by a closed 3-braid admits a unique braid isotopy class of closed 3-braid representatives, with the following exceptions:

- the unknot, which has exactly 3 braid isotopy classes of 3-braid representatives, namely the braid isotopy classes of the closed 3-braids $\sigma_{1}^{\mu} \sigma_{2}^{\tau}$, where $(\mu, \tau) \in$ $\{(1,1),(-1,-1),(1,-1)\}$;

- type $(2, k)$ torus knots, where $k$ is odd and $|k| \neq 1$ which have two braid isotopy classes of 3-braid representatives, namely the conjugacy classes in $B_{3}$ of $\sigma_{1}^{k} \sigma_{2}^{\mu}$, where $\mu= \pm 1$; and

- certain links with braid index 3 which have two 3-braid representatives, related by a positive or negative 3-braid flype.

From this it follows that exactly 4 templates are needed to describe the moves of the MTWS, in the special case where $X_{+}$and $X_{-}$are closed 3-braids which define a knot of braid index at most 3: the two destabilization templates of Figure 2 and the two flype templates of Figure 4 with all weights 1 . In particular, every knot or link which is represented as a closed 3-braid either has a closed braid representative which is unique up to braid isotopy, or it is carried by one of these 4 templates. (We note that, while the 
exchange move template supports knots and links of braid index 3 , it can be replaced by braid isotopy for prime knots and links of braid index at most 3.)

Theorem 1 of [13] asserts that two closed 3-braids are isotopic in the complement of the braid axis if and only if the associated elements in the 3-string braid group are conjugate in $B_{3}$. Using the solution to the conjugacy problem which is given in [14] (it's due to Otto Schreier, 1936) we selected our examples so that their conjugacy classes do not define the unknot or a type $(2, n)$-torus knot, and therefore actually have braid index 3 . We also chose them so that we are certain that the conjugacy classes of $T X_{+}$and $T X_{-}$are actually distinct. Thus the only possibilities, in the topological setting, are that our examples are carried by the positive or negative flype templates. Since we know that transversal knots that are carried by the positive flype template are transversally isotopic, we need to choose the examples so that they are not carried by both flype templates. For that, we turn to [12], where it is shown that the closed braid $\sigma_{1}^{u} \sigma_{2}^{v} \sigma_{1}^{w} \sigma_{2}^{\epsilon}$ admits a flype of sign $\epsilon$ and also a flype of sign $-\epsilon$ if and only if $u=-\epsilon$ or $w=-\epsilon$ or $v=-2 \epsilon$. So we chose our examples to avoid that possibility too.

We have shown that the only possibility, for the particular examples that we chose, is that the transversal isotopy which we assumed exists from $T X_{+}$to $T X_{-}$determines, in the topological setting, a negative flype. Thus, our examples are uniquely carried by a single template in $\mathcal{T}(3,3)$, a negative flyping 3-braid template. And, by Theorem 2 the assumption that $T X_{+}$and $T X_{-}$are transversally isotopic implies this unique template is a transversal template.

We now show that this template isotopy cannot be a transversal isotopy. Here is the reason. A key point about the definition of a template (and this is a very strong aspect of the MTWS) is that for every fixed choice of braiding assignments to the blocks the resulting closed braids represent the same oriented link type $\mathcal{X}$. For example choose the braiding assignments $\sigma_{1}^{3}, \sigma_{2}^{4}, \sigma_{1}^{-5}$ to the blocks $P, R, Q$. This braiding assignment gives a 2-component link $L_{1} \sqcup L_{2}$ which has two distinct isotopy classes of closed 3-braid representatives. If $L_{1}$ is the component associated to the left strand entering the block $P$, then $\beta\left(L_{1}\right)=-1$ and $\beta\left(L_{2}\right)=-3$ before the flype, but after the flype the representative will be $\sigma_{1}^{3} \sigma_{2}^{-1} \sigma_{1}^{-5} \sigma_{2}^{4}$, with $\beta\left(L_{1}\right)=-3$ and $\beta\left(L_{2}\right)=-1$. By Proposition 2.1.2 of [8] a transversal isotopy of a knot/link extends to an ambient transversal isotopy of the 3-sphere. However, any transversal isotopy of $L_{1} \sqcup L_{2}$ must preserve the $\beta$-invariants of the components, so no such transversal isotopy exists, a contradiction of our assumption that $T X_{+}$and $T X_{-}$are transversally isotopic.

\subsection{Future work and some open problems}

Here are some of the ideas and questions that occurred to us in the course of the work in this paper. 
(1) With the help of techniques that use only the machinery of contact structures and characteristic foliations, Etnyre and Honda have proved, in [9], the existence of examples of pairs of transversal cable knots that are of the same knot type, have the same Bennequin number, but are not transversally simple. The type $(2,3)$ cable on a type $(2,3)$ torus knot appears to be an example of this type, although their methods make it difficult to establish this definitively. Their pair of cable knots co-bound an annulus whose characteristic foliation has two homotopically non-trivial Legendrian closed loops. The existence of these loops can be seen as equivalent to the condition that the motion is across a standard annulus (Section 5.8 of [6]) and there are two loops in $G_{\epsilon, \epsilon}$. At this writing it is an open problem to explain their non-explicit examples as explicit examples that use the machinery of the Markov Theorem Without Stabilization.

(2) Either prove or give a counterexample to the conjecture that maximal Bennequin number is always achieved at minimum braid index. In an earlier version of this paper we thought that we had a counterexample, but there was an error in our proof that the braids in question had braid index 6 . In fact Hirose Matsuda showed us that they had braid index 5 and not 6 , so that our counterexample disappeared, and the question remains open.

(3) Characterize the graphs $G_{+,+}$and $G_{-,-}$for an arbitrary transversal clasp annulus, that is without the assumption that we made here that the topological braid index is minimal.

(4) Establish a transversal Markov Theorem Without Stabilization. What should its statement be?

(5) By the work of Giroux [11] there is a one-to-one correspondence between tight contact structures on arbitrary closed, orientable 3-manifolds and open book decompositions of the same manifolds. Noting that every open book decomposition of a closed, orientable 3-manifold can be regarded as a branched covering space of $S^{3}$ with a braid structure, one expects some of the machinery in this paper and [6] to generalize to other 3-manifolds, yielding new connections between the topology and contact geometry of 3-manifolds. In this regard, the question of whether the transverse Markov theorem [15] holds in a more general setting is probably the first question that needs to be answered.

\section{References}

[1] V I Arnol'd, Topological invariants of plane curves and caustics, University Lecture Series 5, American Mathematical Society, Providence, RI (1994) MR1286249 
[2] D Bennequin, Entrelacements et équations de Pfaff, from: "Third Schnepfenried geometry conference, Vol. 1 (Schnepfenried, 1982)”, Astérisque 107, Soc. Math. France, Paris (1983) 87-161 MR753131

[3] J S Birman, E Finkelstein, Studying surfaces via closed braids, J. Knot Theory Ramifications 7 (1998) 267-334 MR1625362

[4] J S Birman, W W Menasco, Studying links via closed braids. III. Classifying links which are closed 3-braids, Pacific J. Math. 161 (1993) 25-113 MR1237139

[5] J S Birman, W W Menasco, On Markov's theorem, J. Knot Theory Ramifications 11 (2002) 295-310 MR1905686

[6] J S Birman, W W Menasco, Stabilization in the braid groups. I. MTWS, Geom. Topol. 10 (2006) 413-540 MR2224463

[7] J S Birman, N C Wrinkle, On transversally simple knots, J. Differential Geom. 55 (2000) 325-354 MR1847313

[8] Y Eliashberg, Legendrian and transversal knots in tight contact 3-manifolds, from: "Topological methods in modern mathematics (Stony Brook, NY, 1991)", Publish or Perish, Houston, TX (1993) 171-193 MR1215964

[9] J B Etnyre, K Honda, Cabling and transverse simplicity, Ann. of Math. (2) 162 (2005) 1305-1333 MR2179731

[10] D Fuchs, S Tabachnikov, Invariants of Legendrian and transverse knots in the standard contact space, Topology 36 (1997) 1025-1053 MR1445553

[11] E Giroux, Géométrie de Contact:de la Dimension Trois vers les Dimensions Supérieures arXiv:math.GT/0305129

[12] K H Ko, S J Lee, Flypes of closed 3-braids in the standard contact space, J. Korean Math. Soc. 36 (1999) 51-71 MR1669133

[13] H R Morton, Infinitely many fibred knots having the same Alexander polynomial, Topology 17 (1978) 101-104 MR486796

[14] K Murasugi, On closed 3-braids, American Mathematical Society, Providence, R.I. (1974) MR0356023

[15] S Y Orevkov, V V Shevchishin, Markov theorem for transversal links, J. Knot Theory Ramifications 12 (2003) 905-913 MR2017961

[16] N Wrinkle, PhD thesis, Columbia University (2002)

Department of Mathematics, Barnard College, Columbia University

2990 Broadway, New York, NY 10027, USA

Department of Mathematics, University at Buffalo, Buffalo, NY 14260, USA

jb@math.columbia.edu, menasco@math.buffalo.edu

Proposed: Rob Kirby

Seconded: David Gabai, Cameron Gordon
Received: 24 June 2005

Accepted: 28 June 2006 